\title{
Quantitative functional calculus in Sobolev spaces
}

\author{
Carlo Morosi* and Livio Pizzocchero
}

(Communicated by Jürgen Appell)

2000 Mathematics Subject Classification. 46E35, 26D10, 47A60.

Keywords and phrases. Sobolev spaces, inequalities, Nemytskij operators.

\begin{abstract}
In the framework of Sobolev (Bessel potential) spaces $H^{n}\left(\mathbf{R}^{d}, \mathbf{R}\right.$ or $\left.\mathbf{C}\right)$, we consider the nonlinear Nemytskij operator sending a function $x \in \mathbf{R}^{d} \mapsto f(x)$ into a composite function $x \in \mathbf{R}^{d} \mapsto G(f(x), x)$. Assuming sufficient smoothness for $G$, we give a "tame" bound on the $H^{n}$ norm of this composite function in terms of a linear function of the $H^{n}$ norm of $f$, with a coefficient depending on $G$ and on the $H^{a}$ norm of $f$, for all integers $n, a, d$ with $a>d / 2$. In comparison with previous results on this subject, our bound is fully explicit, allowing to estimate quantitatively the $H^{n}$ norm of the function $x \mapsto G(f(x), x)$. When applied to the case $G(f(x), x)=f^{2}(x)$, this bound agrees with a previous result of ours on the pointwise product of functions in Sobolev spaces.
\end{abstract}

\section{Introduction}

Functional calculus in Sobolev spaces has been extensively discussed, from the classic papers [1], [4] to the recent book [19]. These and the other works of our knowledge have been mainly devoted to the well definedness of nonlinear composition operators (Nemytskij operators), and

${ }^{*}$ Corresponding author. 
to the qualitative structure of the norm estimates for these operators. On the contrary, in this paper we are interested in giving fully quantitative norm estimates for the Nemytskij operators, for the special case of $L^{2}$ type Sobolev spaces, including evaluation of all numerical constants involved therein; the spirit is the same as in the previous work [18] on the pointwise product of two functions in a Sobolev space, whose results imply in an elementary way estimates on the operators of composition with polynomials. Here we will infer estimates for much more general Nemytskij operators, covering essentially the composition with any sufficiently derivable mapping. When the present, general results are applied to polynomial mappings, in the quadratic case they reproduce the upper bounds arising from [18] (whose reliability was also tested in the cited work, by comparison with the lower bounds obtained from appropriate trial functions); for polynomial Nemytskij operators of degree $\geq 3$, the upper bounds derived in the present framework are even more efficient than the elementary iteration of the estimates in [18].

The Sobolev spaces we consider in this paper are the $L^{2}$ type Sobolev (Bessel potential) spaces $H^{n}\left(\mathbf{R}^{d}, \mathbf{C}\right)$, or their real analogues $H^{n}\left(\mathbf{R}^{d}, \mathbf{R}\right)$, with the corresponding norms \|\|$_{n}:=\|\|_{H^{n}}$ (see [2] [13] or Section 2); the attention is restricted to the case of integer order $n$.

Before describing our results on Nemytskij operators, let us fix some notations. Consider a function $G: \mathcal{B} \times \mathbf{R}^{d} \rightarrow \mathbf{R}$ or $\mathbf{C}$, where $\mathcal{B}$ is an interval of $\mathbf{R}$ or a ball of $\mathbf{C}$, say, with center 0 ; if $f: \mathbf{R}^{d} \mapsto \mathcal{B}$ and $\chi: \mathbf{R}^{d} \mapsto \mathbf{R}^{d}$, one can define the composition $G(f, \chi): \mathbf{R}^{d} \mapsto \mathbf{R}$ or $\mathbf{C}, x \mapsto G(f(x), \chi(x))$. We choose for $\chi$ the identity mapping $\mathrm{x}: \mathbf{R}^{d} \rightarrow \mathbf{R}^{d}, x=\left(x_{1}, \ldots, x_{d}\right) \mapsto x$; thus

$$
G(f, \mathrm{x}): \mathbf{R}^{d} \mapsto \mathbf{R} \text { or } \mathbf{C}, \quad x \mapsto G(f(x), x) .
$$

The (generally nonlinear) mapping

$$
f \mapsto G(f, \mathrm{x})
$$

will be called the Nemytskij operator associated to $G$. Its domain is the set of functions (modulo equality almost everywhere) $f: \mathbf{R}^{d} \rightarrow \mathcal{B}, x \mapsto f(x)$, and the codomain is made of functions $\mathbf{R}^{d} \mapsto \mathbf{R}$ or $\mathbf{C}$ (in the sequel, the expression " $G(f, \mathbf{x})$ is defined" will always be employed to mean that a function $f$ on $\mathbf{R}^{d}$ takes values in $\mathcal{B}$ ).

The classical problem of functional calculus in Sobolev spaces is to prove that $G(f, \mathrm{x})$ is in a Sobolev space, and to estimate its norm, when $G$ is sufficiently smooth and $f$ is in a related Sobolev space. To discuss this topic one can fix a base integer $a>d / 2$, so that functions in $H^{a}$ be bounded and continuous (in applications, $a$ is often chosen to be the smallest integer $>d / 2$ ); a second integer $n$ runs freely over $\mathbf{N}$, labelling the scale of all $H^{n}$ 
spaces. As well known, if $G$ satisfies suitable conditions of smoothness and boundedness, $G(0, \mathrm{x})$ is in $H^{n}$ and $f$ is in the intersection of $H^{n}$ with a ball of $H^{a}$, then $G(f, \mathrm{x})$ is defined, it belongs to $H^{n}$ and

$$
\|G(f, \mathrm{x})-G(0, \mathrm{x})\|_{n} \leq \Upsilon_{n a d}\left(G,\|f\|_{a}\right)\|f\|_{n} ;
$$

the r.h.s. in this inequality is a linear function of $\|f\|_{n}$, with a coefficient depending on $\|f\|_{a}$ in a way determined by $n, a, d$ and by the function $G$.

Our aim is to provide an explicit expression for the function $\Upsilon_{n a d}$ ruling this dependence: this will be given in Propositions 2.4 and 2.7, which are the main results of the paper. One feature of the expression we will obtain is that it does not depend dramatically on the dimension $d$ (differently from the bounds frequently appearing in similar situations, when the indices of derivatives are manipulated too naively); this result is obtained by systematic use of tensorial methods to express the derivatives of any order of functions on $\mathbf{R}^{d}$ and their composition with $G$. Another feature of our result, already stressed, is that it contains, as a subcase, the outcomes of [18] on the case $G(f, \mathrm{x})=f^{2}$, the pointwise product of $f$ by itself (estimates on pointwise product were derived in [18] using the Fourier transform, instead of the present tensor approach, as an alternative tool to deal efficiently with the $d$-dependence).

In Eq. (1.3) one recognises the structure of the so-called "tame" estimates, forming the basis of the Nash-Moser implicit function theorem [9]. More precisely, we have a "tame estimate of order zero" (an estimate of general order $r$ would give the $n$-th norm of a nonlinear operator on $f$ in terms of a linear function of $\|f\|_{n+r}$, with a coefficient depending on $\|f\|_{a}$ for some fixed order a). Such zero order estimates are used in [16] in relation to semilinear evolution equations on the intersection $\cap_{n=0}^{\infty} H^{n}$; the results of the present paper allow quantitative estimates on the existence times for the solutions, when the nonlinear part of the evolution equation is a Nemytskij operator.

Let us illustrate the organisation of the paper. In Section 2 we describe the main results, i.e., the expression of $\Upsilon_{\text {nad }}$ in the case of real and complex Sobolev spaces; this requires some preliminaries on the tensor formalism and the definition of some "universal" polynomials $P_{m}(m=1,2,3, \ldots)$ in terms of generating relations. The rest of the paper is devoted to the proofs. In Section 3 we write a Leibnitz rule for the derivatives of tensor fields on $\mathbf{R}^{d}$, and present a generalized Faà di Bruno formula for the derivatives of any order of $G(f, \mathrm{x})$; both constructions rely on symmetrised tensor products, which are conveniently discussed. In Section 4 we prove the main properties of the polynomials $P_{m}$. In Section 5 we discuss some functional spaces of tensor fields and the Hausdorff-Young inequality for the Fourier transform of 
tensors; also, we propose estimates for the constants in a Gagliardo type and an Adams-Frazier type inequality. In Section 6 we use these tools to derive the expression of $\Upsilon_{\text {nad }}$ presented in Section 2. Finally, in the Appendix we prove some technical statements on tensor norms and the symmetric tensor calculus, which are necessary to derive the main results of the paper.

\section{Description of the results}

Throughout the paper $\mathbf{N}:=\{0,1,2, \ldots\}$ and $\mathbf{N}_{0}:=\{1,2,3, \ldots\} ; d \in \mathbf{N}_{0}$ is a fixed space dimension.

Tensor notations and Sobolev spaces. For our purposes, it is convenient to introduce some tensor representations for expressions with indices, and some related spaces of tensor-valued functions. This setting will simplify the exposition of the results and the subsequent proofs.

For $m \in \mathbf{N}_{0}$, we indicate with $\otimes^{m} \mathbf{C}^{d}$ the tensor product of $m$ copies of $\mathbf{C}^{d}$; this will be identified with the complex vector space of families

$$
T=\left(T_{\lambda}\right)_{\lambda=\left(\lambda_{1}, \ldots, \lambda_{m}\right) \in\{1, \ldots, d\}^{m}},
$$

where $T_{\lambda} \in \mathbf{C}$ for all $\lambda$; any such $T$ will be called a complex tensor of order $m$. The norm of $T$ is

$$
|T|:=\sqrt{\sum_{\lambda}\left|T_{\lambda}\right|^{2}} .
$$

In the sequel, we always intend $\otimes^{0} \mathbf{C}^{d}:=\mathbf{C}$ (as customary for tensor products). Let $p \in[1,+\infty]$; then, $L^{p}\left(\mathbf{R}^{d}, \otimes^{m} \mathbf{C}^{d}\right)$ is the space of measurable functions (modulo equality almost everywhere)

$$
T: \mathbf{R}^{d} \rightarrow \otimes^{m} \mathbf{C}^{d}, \quad x \mapsto T(x)=\left(T_{\lambda}(x)\right)
$$

such that the function

$$
|T|: \mathbf{R}^{d} \mapsto[0,+\infty), \quad x \mapsto|T(x)|=\sqrt{\sum_{\lambda}\left|T_{\lambda}(x)\right|^{2}}
$$

is in $L^{p}\left(\mathbf{R}^{d}, \mathbf{R}\right)$. Obviously enough, this space is identified with the space of families $T=\left(T_{\lambda}\right)$ where $T_{\lambda} \in L^{p}\left(\mathbf{R}^{d}, \mathbf{C}\right)$ for each $\lambda \in\{1, \ldots, d\}^{m}$. The $L^{p}$ norm of a function $T$ as above is

$$
\|T\|_{L^{p}}:=\||T|\|_{L^{p}} .
$$


We use the standard notations $\mathcal{D}\left(\mathbf{R}^{d}, \mathbf{C}\right), \mathcal{D}^{\prime}\left(\mathbf{R}^{d}, \mathbf{C}\right)$ for the space of smooth, compactly supported functions $\mathbf{R}^{d} \mapsto \mathbf{C}$ and the space of distributions. More generally, $\mathcal{D}\left(\mathbf{R}^{d}, \otimes^{m} \mathbf{C}^{d}\right)$ is the space of smooth, compactly supported functions $\Phi: \mathbf{R}^{d} \rightarrow \otimes^{m} \mathbf{C}^{d}$, which are identified with the families $\Phi=$ $\left(\Phi_{\lambda}\right)_{\lambda \in\{1, \ldots, d\}^{m}}$ with each component in $\mathcal{D}\left(\mathbf{R}^{d}, \mathbf{C}\right)$. We define $\mathcal{D}^{\prime}\left(\mathbf{R}^{d}, \otimes^{m} \mathbf{C}^{d}\right)$ to be the space of families $T=\left(T_{\lambda}\right)_{\lambda \in\{1, \ldots, d\}^{m}}$ with each component in $\mathcal{D}^{\prime}\left(\mathbf{R}^{d}, \mathbf{C}\right)$.

Let $f \in C^{m}\left(\mathbf{R}^{d}, \mathbf{C}\right)$, or $f \in \mathcal{D}^{\prime}\left(\mathbf{R}^{d}, \mathbf{C}\right)$. We put

$$
\nabla^{m} f:=\left(\partial_{\lambda} f\right)_{\lambda=\left(\lambda_{1}, \ldots, \lambda_{m}\right) \in\{1, \ldots, d\}^{m}}
$$

where $\partial_{\lambda}:=\partial_{\lambda_{1}} \ldots \partial_{\lambda_{m}}$ ( $\partial_{\lambda_{i}}$ the partial derivative in the $\lambda_{i}$-th direction) and the derivatives are intended in the ordinary or in the distributional sense; we also intend $\nabla^{0} f:=f$.

In the above tensor style, the $L^{2}$ type space of complex Bessel potentials of integer order $n \in \mathbf{N}$ can be defined setting

$$
H^{n}\left(\mathbf{R}^{d}, \mathbf{C}\right):=\left\{f \in \mathcal{D}^{\prime}\left(\mathbf{R}^{d}, \mathbf{C}\right) \mid \nabla^{m} f \in L^{2}\left(\mathbf{R}^{d}, \otimes^{m} \mathbf{C}^{d}\right) \forall m \in\{0, \ldots, n\}\right\} ;
$$

this space carries the (Hilbertian) norm

$$
\|f\|_{n}:=\sqrt{\sum_{m=0}^{n}\left(\begin{array}{c}
n \\
m
\end{array}\right)\left\|\nabla^{m} f\right\|_{L^{2}}^{2}} .
$$

Definitions (2.7) and (2.8) will be our standards throughout the paper. In the integer case we consider, they coincide exactly with the conventional definition of $H^{n}$ [2] [13] in terms of the operator $\sqrt{1-\Delta}^{n}$, a power of 1 minus the Laplacian $\Delta$ constructed via the Fourier tranform of tempered distributions. In particular, the norm $\|f\|_{n}$ in Eq. (2.8) equals $\left\|\sqrt{1-\Delta}^{n} f\right\|_{L^{2}}$, which is the standard norm of our previous works [17], $[18]\left({ }^{\dagger}\right)$.

For $m \in \mathbf{N}$, the tensor power $\otimes^{m} \mathbf{R}^{d}$, the spaces $L^{p}\left(\mathbf{R}^{d}, \otimes^{m} \mathbf{R}^{d}\right)$ and their norms are constructed in an obvious way, with $\mathbf{R}$ replacing $\mathbf{C}$ everywhere. Similarly, we can introduce $\nabla^{m} f$ when $f$ is a real-valued function or distribution on $\mathbf{R}^{d}$; this allows to define $H^{n}\left(\mathbf{R}^{d}, \mathbf{R}\right)$ and its norm writing the analogues of Eqs. (2.7)-(2.8).

It should be noted that, due to the permutability of partial derivatives, the derivatives $\nabla^{m} f$ of a real or complex function $f$ take values in the space of symmetric tensors, on which we will return later from a more systematic viewpoint.

\footnotetext{
${ }^{\dagger}$ The representation of this norm via multiindices given in [18] for the integer case is just a rephrasement of Eq. (2.8), less efficient for our present purposes.
} 
Useful constants and notations. For any integer $a>d / 2$, we put

$$
S_{a d}:=\frac{1}{(4 \pi)^{d / 4}} \sqrt{\frac{\Gamma(a-d / 2)}{\Gamma(a)}},
$$

$\Gamma$ denoting the factorial function. This is the sharp constant in the imbedding inequality of $H^{a}\left(\mathbf{R}^{d}, \mathbf{R}\right.$ or $\left.\mathbf{C}\right)$ into $L^{\infty}\left(\mathbf{R}^{d}, \mathbf{R}\right.$ or $\left.\mathbf{C}\right)$; thus

$$
\|\|_{L^{\infty}} \leq S_{a d}\|\|_{a}
$$

and $S_{a d}$ is the minimum constant for which this occurs [17]. For $a$ as before and $r \in(0,+\infty]$, we put

$$
H_{r}^{a}\left(\mathbf{R}^{d}, \mathbf{R} \text { or } \mathbf{C}\right):=\left\{f \in H^{a}\left(\mathbf{R}^{d}, \mathbf{R} \text { or } \mathbf{C}\right) \mid S_{a d}\|f\|_{a}<r\right\}
$$

(intending this to be the whole space $H^{a}$, if $r=+\infty$ ); then

$$
f \in H_{r}^{a}\left(\mathbf{R}^{d}, \mathbf{R} \text { or } \mathbf{C}\right) \Longrightarrow\|f\|_{L^{\infty}}<r
$$

(all this can be extended to the case of a noninteger $a>d / 2$, but here we are not considering fractional Sobolev spaces).

Let $E:[0,+\infty) \rightarrow\left[e^{-1 / e},+\infty\right)$ be defined by

$$
E(s):=s^{s} \quad \text { for } s \in(0,+\infty), \quad E(0):=\lim _{s \rightarrow 0^{+}} E(s)=1 .
$$

Using this function, we define

$$
U_{m j d}:=\left(\frac{E(1 / 2-(j-1) /(2 m))}{E(1 / 2+(j-1) /(2 m))}\right)^{d / 2}\left(\frac{E(1 /(2 m))}{E(1-1 /(2 m))}\right)^{(j-1) d / 2}
$$

for all integers $m, j$ with $1 \leq j \leq m$. It should be noted that

$$
U_{m j d} \leq 1
$$

for $m, j$ as above; in fact, $U_{m j d}$ is a product of factors of the form $E(s) / E(1-s)$, with $s \in[0,1 / 2]$, raised to power $d / 2$; each one of these factors is $\leq 1$. We will show how the constant $U_{m j d}$ arises in an AdamsFrazier type inequality for products of derivatives (see Proposition 5.3).

Defining the "universal" polynomials $\boldsymbol{P}_{\boldsymbol{m}}$. As anticipated in the Introduction, these polynomials will be employed to express the main results on Nemytskij operators; we will give some equivalent descriptions for them, via the following Lemma, to be proved in Section 4. 
Lemma 2.1. Let $m \in \boldsymbol{N}_{0}$. Then, there is a unique real polynomial $P_{m}\left(\left(\nu_{j \ell}\right), \rho\right)$, in a set of variables

$$
\nu_{j \ell}, \rho \quad(1 \leq j \leq m, 0 \leq \ell \leq m-j),
$$

such that for each $C^{m}$ function $F: \boldsymbol{R}^{2} \mapsto \boldsymbol{R},(u, \xi) \mapsto F(u, \xi)$ it is

$$
\frac{d^{m}}{d \xi^{m}} F\left(e^{\xi}, \xi\right)=P_{m}\left(\nu_{j \ell}=\frac{\partial^{j+\ell} F}{\partial u^{j} \partial \xi^{\ell}}\left(e^{\xi}, \xi\right), \rho=e^{\xi}\right)+\frac{\partial^{m} F}{\partial \xi^{m}}\left(e^{\xi}, \xi\right)
$$

This polynomial has the form

$$
P_{m}\left(\left(\nu_{j \ell}\right), \rho\right)=\sum_{\substack{1 \leq j \leq m \\ 0 \leq \ell \leq m-j}} P_{m j \ell} \nu_{j \ell} \rho^{j}
$$

where the $P_{m j \ell}$ are positive, integer coefficients; these have the equivalent characterizations

$$
\begin{gathered}
P_{m j \ell}=\left.\frac{d^{m}}{d \xi^{m}}\right|_{\xi=0} \frac{\left(e^{\xi}-1\right)^{j} \xi^{\ell}}{j ! \ell !}, \\
P_{m j \ell}=\frac{1}{j !}\left(\begin{array}{c}
m \\
\ell
\end{array}\right) \sum_{s=0}^{j}(-1)^{j-s}\left(\begin{array}{c}
j \\
s
\end{array}\right) s^{m-\ell},
\end{gathered}
$$

and can be described as the solutions of the recursive equations

$$
\begin{gathered}
P_{110}=1, \quad P_{100}=0 \\
P_{m+1, j \ell}=P_{m j, \ell-1}+P_{m, j-1, \ell}+j P_{m j \ell}+\delta_{j 1} \delta_{\ell m} \\
\text { for } m \geq 1,1 \leq j \leq m+1,0 \leq \ell \leq m+1-j
\end{gathered}
$$

(intending $P_{m j,-1}:=P_{m, m+1, \ell}:=0 ; \delta_{i j}$ is the Kronecker symbol).

The defining relation (2.17) for the polynomials $P_{m}$ point out their link with the derivation of composite functions, a topic which has obvious connections with the analysis of Nemytskij operators; Eq. (2.17) also allows very simple derivations of some identities for these polynomials (see, e.g., Eq. (2.56)).

The automatic computation of the polynomials $P_{m}$ can be easily performed, implementing Eqs. (2.17) or (2.19) on any package for symbolic manipulation, or the purely numerical relations (2.20) or (2.21) on any 


\begin{aligned} & \hline \hline Table of low order polynomials $\boldsymbol{P}_{\boldsymbol{m}} \\ &$\hline \hline$P_{1}\left(\left(\nu_{j \ell}\right), \rho\right)= \nu_{10} \rho \\ & P_{2}\left(\left(\nu_{j \ell}\right), \rho\right)= \nu_{20} \rho^{2}+\left(\nu_{10}+2 \nu_{11}\right) \rho \\ & P_{3}\left(\left(\nu_{j \ell}\right), \rho\right)= \nu_{30} \rho^{3}+\left(3 \nu_{20}+3 \nu_{21}\right) \rho^{2}+\left(\nu_{10}+3 \nu_{11}+3 \nu_{12}\right) \rho \\ & P_{4}\left(\left(\nu_{j \ell}\right), \rho\right)= \nu_{40} \rho^{4}+\left(6 \nu_{30}+4 \nu_{31}\right) \rho^{3}+\left(7 \nu_{20}+12 \nu_{21}+6 \nu_{22}\right) \rho^{2} \\ &+\left(\nu_{10}+4 \nu_{11}+6 \nu_{12}+4 \nu_{13}\right) \rho \\ & P_{5}\left(\left(\nu_{j \ell}\right), \rho\right)= \nu_{50} \rho^{5}+\left(10 \nu_{40}+5 \nu_{41}\right) \rho^{4}+\left(25 \nu_{30}+30 \nu_{31}+10 \nu_{32}\right) \rho^{3} \\ &+\left(15 \nu_{20}+35 \nu_{21}+30 \nu_{22}+10 \nu_{23}\right) \rho^{2} \\ &+\left(\nu_{10}+5 \nu_{11}+10 \nu_{12}+10 \nu_{13}+5 \nu_{14}\right) \rho \\ & P_{6}\left(\left(\nu_{j \ell}\right), \rho\right)= \nu_{60} \rho^{6}+\left(15 \nu_{50}+6 \nu_{51}\right) \rho^{5}+\left(65 \nu_{40}+60 \nu_{41}+15 \nu_{42}\right) \rho^{4} \\ &+\left(90 \nu_{30}+150 \nu_{31}+90 \nu_{32}+20 \nu_{33}\right) \rho^{3} \\ &+\left(31 \nu_{20}+90 \nu_{21}+105 \nu_{22}+60 \nu_{23}+15 \nu_{24}\right) \rho^{2} \\ &+\left(\nu_{10}+6 \nu_{11}+15 \nu_{12}+20 \nu_{13}+15 \nu_{14}+6 \nu_{15}\right) \rho \\ &$\hline \hline\end{aligned}

system capable to deal with integer numbers. The polynomials $P_{m}$ for $1 \leq m \leq 6$ are reported in a separate Table.

There is a strict relation between the coefficients $P_{m j \ell}$ and the Stirling numbers. Let us recall that the Stirling number of the second kind $\mathcal{S}_{m}^{j}$ is defined to be the number of partitions of a set of $m$ elements into $j$ nonempty subsets; comparing some standard results on these numbers [5] with anyone of Eqs. (2.19-2.21), it is found that

$$
P_{m j \ell}=\left(\begin{array}{c}
m \\
\ell
\end{array}\right) \mathcal{S}_{m-\ell}^{j} \quad(1 \leq j \leq m, 0 \leq \ell \leq m-j) .
$$

(In particular, $P_{m j 0}=\mathcal{S}_{m}^{j}$; these are the only coefficients involved in the derivative (2.17) in the case of a $\xi$-independent function $F(u)$, which is considered in [5]. The $P_{m j 0}$ will also be the only coefficients appearing in our estimates on Nemystkij operators $f \mapsto G(f)$ with an $x$-independent $G)$.

Nemytskij operators in the real case. Let us consider a function

$$
G:(-r, r) \times \mathbf{R}^{d} \rightarrow \mathbf{R}, \quad(u, x)=\left(u, x_{1}, \ldots, x_{d}\right) \mapsto G(u, x)
$$

with $r \in(0,+\infty]$. Concerning partial derivatives in the above variables, we use the notations

$$
\partial^{j}:=\frac{\partial^{j}}{\partial u^{j}} \quad(j \in \mathbf{N})
$$


$\partial_{\lambda}:=\partial_{\lambda_{1}} \ldots \partial_{\lambda_{\ell}}:=\frac{\partial}{\partial x_{\lambda_{1}}} \ldots \frac{\partial}{\partial x_{\lambda_{\ell}}} \quad\left(\ell \in \mathbf{N}, \lambda=\left(\lambda_{1}, \ldots, \lambda_{\ell}\right) \in\{1, \ldots, d\}^{\ell}\right)$.

For $j, \ell \in \mathbf{N}$ and $G$ of class $C^{j+\ell}$, the family of partial derivatives

$$
\partial^{j} \nabla^{\ell} G:=\left(\partial^{j} \partial_{\lambda} G\right)_{\lambda \in\{1, \ldots, d\}^{\ell}}
$$

can be seen as a continuous function $\partial^{j} \nabla^{\ell} G:(-r, r) \times \mathbf{R}^{d} \rightarrow \otimes^{\ell} \mathbf{R}^{d}$.

Definition 2.2. Given the map $G:(-r, r) \times \mathbf{R}^{d} \rightarrow \mathbf{R}$, we put

$$
\begin{gathered}
b_{m}(G, \rho):=\sup _{\substack{u \in[-\rho, \rho] \backslash\{0\} \\
x \in \mathbf{R}^{d}}}\left|\frac{\nabla^{m} G(u, x)-\nabla^{m} G(0, x)}{u}\right| \quad(\rho \in(0, r)), \\
(2.28) \quad \sigma_{j \ell}(G, \rho):=\sup _{\substack{u \in[-\rho, \rho] \\
x \in \mathbf{R}^{d}}}\left|\partial^{j} \nabla^{\ell} G(u, x)\right| \quad(\rho \in[0, r))
\end{gathered}
$$

if $m, j, \ell$ are integers and $G$ is $C^{m}$ or $C^{j+\ell}$, respectively (the indicated suprema being possibly infinite). Also, we put

$$
\begin{aligned}
& \beta_{m d}(G, \rho):=P_{m}\left(\nu_{j \ell}=\left(1-\frac{\ell}{m}\right) U_{m-\ell, j d} \frac{\sigma_{j \ell}(G, \rho)}{\rho}, \rho\right) \quad(\rho \in(0, r)), \\
& \quad b_{m d}(G, \rho):=P_{m}\left(\nu_{j \ell}=\frac{\ell}{m} U_{m-\ell, j d} \frac{\sigma_{j \ell}(G, \rho)}{\rho}, \rho\right) \quad(\rho \in(0, r)),
\end{aligned}
$$

if $m \geq 1$ is an integer and $G$ is $C^{m}$ (the r.h.s. of the last two equations being possibly $+\infty$, when infinite suprema $\sigma_{j \ell}(G, \rho)$ are involved; in any case, it must be understood that $\nu_{j 0}=0$ in Eq. (2.30)). Here, $P_{m}$ is the polynomial defined by Lemma 2.1 and the constants $U_{m-\ell, j d}$ are defined according to Eq. (2.14).

Of course $b_{m}(G, \rho) \leq b_{m}\left(G, \rho_{1}\right), \sigma_{j \ell}(G, \rho) \leq \sigma_{j \ell}\left(G, \rho_{1}\right)$ for $\rho \leq \rho_{1}$; thus, if anyone of these suprema is finite for some radius $\rho_{1}$, it is also finite for all radii $\rho \leq \rho_{1}$. For uniformity of language, it is convenient to define $b_{m}(G, \rho)$, $\beta_{m d}(G, \rho)$ and $b_{m d}(G, \rho)$ at $\rho=0$ also.

If $b_{m}(G, \rho)$ is finite for $\rho$ in a right neighborhood of zero, we will make the natural choice $b_{m}(G, 0):=\lim _{\rho \rightarrow 0^{+}} b_{m}(G, \rho)$. By monotonicity this limit exists; it is finite and nonnegative. 
We will also intend $\beta_{m d}(G, 0):=\lim _{\rho \rightarrow 0^{+}} \beta_{m d}(G, \rho), \quad b_{m d}(G, 0):=$ $\lim _{\rho \rightarrow 0^{+}} b_{m d}(G, \rho)$, if the r.h.s. are finite for $\rho$ close to zero. Again, the limits exist finite and $\geq 0$ : in fact, in the $\nu_{j \ell}$ arguments of $P_{m}$ the terms $\sigma_{j \ell}(G, \rho)$ admit finite limits by monotonicity, and the factors $1 / \rho$ are cancelled out by the powers $\rho^{j}(j \geq 1)$, see Eq. (2.18).

Definition 2.3. Let $n \in \mathbf{N}$. A map $G$ as above is said to have the $\Pi^{n}$ property if:

(i) $G$ is $C^{n}$;

(ii) $b_{m}(G, \rho)<+\infty$ for $0 \leq m \leq n$ and all $\rho \in(0, r)$; $\sigma_{j \ell}(G, \rho)<+\infty$ for $1 \leq j \leq n, 0 \leq \ell \leq n-j$ and all $\rho \in[0, r)$

(iii) the functions $b_{m}(G,),. \sigma_{j \ell}(G,):.[0, r) \rightarrow[0,+\infty)$ are continuous for all $m, j, \ell$ as in ii).

Of course, iii) implies continuity of the functions $b_{m d}(G,),. \beta_{m d}(G,$.$) :$ $[0, r) \rightarrow[0,+\infty)$ for $1 \leq m \leq n$. Let us recall the definitions (2.9) of the constant $S_{a d}$ and $(2.11)$ of the ball $H_{r}^{a}\left(\mathbf{R}^{d}, \mathbf{R}\right)$, where $a>d / 2$; for $G:(-r, r) \times \mathbf{R}^{d} \rightarrow \mathbf{R}$ and $f \in H_{r}^{a}\left(\mathbf{R}^{d}, \mathbf{R}\right), G(f, \mathbf{x})$ is defined on the grounds of Eq. (2.12).

With the above terminology, the main result on real Nemystkij operators is the following.

Proposition 2.4. Let $n, a \in \boldsymbol{N}$ and $a>d / 2$. Consider:

(i) a function $G:(-r, r) \times \boldsymbol{R}^{d} \rightarrow \boldsymbol{R}$ with the $\Pi^{n}$ property, such that $G(0, \mathrm{x}): x \mapsto G(0, x)$ is in $H^{n}\left(\boldsymbol{R}^{d}, \boldsymbol{R}\right)$;

(ii) a function $f \in H^{n}\left(\boldsymbol{R}^{d}, \boldsymbol{R}\right) \cap H_{r}^{a}\left(\boldsymbol{R}^{d}, \boldsymbol{R}\right)$.

Then $G(f, \mathrm{x}) \in H^{n}\left(\boldsymbol{R}^{d}, \boldsymbol{R}\right)$ and

$\|G(f, \mathrm{x})-G(0, \mathrm{x})\|_{n} \leq \gamma_{n d}\left(G, S_{a d}\|f\|_{a}\right)\|f\|_{n}+c_{n d}\left(G, S_{a d}\|f\|_{a}\right)\|f\|_{L^{2}}$

where, for any $\rho \in[0, r)$,

$$
\gamma_{n d}(G, \rho):=\max \left\{b_{0}(G, \rho), \beta_{m d}(G, \rho)(m=1, \ldots, n)\right\},
$$

$$
c_{n d}(G, \rho):=\sqrt{\sum_{m=1}^{n}\left(\begin{array}{c}
n \\
m
\end{array}\right)\left(b_{m d}(G, \rho)+b_{m}(G, \rho)\right)^{2}}
$$

(intending these to be $b_{0}(G, \rho)$ and 0 , respectively, for $n=0$ ).

Eq. (2.31) implies the weaker bound

$$
\|G(f, \mathrm{x})-G(0, \mathrm{x})\|_{n} \leq\left(\gamma_{n d}\left(G, S_{a d}\|f\|_{a}\right)+c_{n d}\left(G, S_{a d}\|f\|_{a}\right)\right)\|f\|_{n} .
$$


Remarks. (i) Of course, the coefficient of $\|f\|_{n}$ in the r.h.s. of Eq. (2.34) is the term denoted with $\Upsilon_{\text {nad }}\left(G,\|f\|_{a}\right)$ in the Introduction.

(ii) We recall that $\beta_{m d}$ and $b_{m d}$ depend on the constants $U_{m-\ell, j d} \leq 1$; weaker but simpler estimates can be obtained replacing systematically those constants with one.

(iii) The previous results become simpler if $G$ is $x$-independent: $G(u, x)=$ $G(u)$; this case is frequently considered in the investigation of Nemytskij operators and will be discussed in more detail at the end of this Section, also giving two examples. A main feature of this case is that $c_{n d}(G, \rho)=0$ for all $n, \rho$.

Nemytskij operators in the complex case. From now on $B_{\mathbf{C}}(0, r)$ denotes the open ball in $\mathbf{C}$ of center 0 and radius $r$, and $\bar{B}_{\mathbf{C}}(0, r)$ its closure. We consider a function

$$
G: B_{\mathbf{C}}(0, r) \times \mathbf{R}^{d} \rightarrow \mathbf{C}, \quad(z, x)=\left(z, x_{1}, \ldots, x_{d}\right) \mapsto G(z, x)
$$

with $r \in(0,+\infty]$. For partial derivatives with respect to the complex variable, we use the notations

$$
\partial^{h} \bar{\partial}^{k}:=\frac{\partial^{h}}{\partial z^{h}} \frac{\partial^{k}}{\partial \bar{z}^{k}} \quad(h, k \in \mathbf{N}) ;
$$

we write $\partial_{\lambda}$, as in Eq. (2.25), for the derivatives w.r.t. the $x$ variables. We say that $G$ is $C^{n}$ if all partial derivatives of $G$ of order $\leq n$ w.r.t. $z, \bar{z}$ and $\left(x_{1}, \ldots, x_{d}\right)$ exist and are continuous, everywhere on its domain. We put

$$
\partial^{h} \bar{\partial}^{k} \nabla^{\ell} G:=\left(\partial^{h} \bar{\partial}^{k} \partial_{\lambda} G\right)_{\lambda \in\{1, \ldots, d\}^{\ell}}
$$

for $h, k, \ell \in \mathbf{N}$ and $G$ of class $C^{h+k+\ell}$; this can be regarded as a function $B_{\mathbf{C}}(0, r) \times \mathbf{R}^{d} \rightarrow \otimes^{\ell} \mathbf{C}^{d}$.

Definition 2.5. Given the function $G: B_{\mathbf{C}}(0, r) \times \mathbf{R}^{d} \rightarrow \mathbf{C}$, we put (2.38)

$$
\begin{aligned}
& b_{m}(G, \rho):=\sup _{\substack{z \in \bar{B}_{\mathbf{C}}(0, \rho) \backslash\{0\} \\
x \in \mathbf{R}^{d}}}\left|\frac{\nabla^{m} G(z, x)-\nabla^{m} G(0, x)}{z}\right| \quad(\rho \in(0, r)), \\
& \sigma_{h k \ell}(G, \rho):=\sup _{\substack{z \in \bar{B}_{\mathbf{C}}(0, \rho) \\
x \in \mathbf{R}^{d}}}\left|\partial^{h} \bar{\partial}^{k} \nabla^{\ell} G(z, x)\right| \quad(\rho \in[0, r))
\end{aligned}
$$




$$
\sigma_{j \ell}(G, \rho):=\sum_{h=0}^{j}\left(\begin{array}{l}
j \\
h
\end{array}\right) \sigma_{h, j-h, \ell}(G, \rho) \quad(\rho \in[0, r))
$$

for $m, h, k, j, \ell$ integers and $G$ of class $C^{m}, C^{h+k+\ell}$ and $C^{j+\ell}$, respectively (these quantities being possibly infinite).

For $m \geq 1$ integer and $\rho \in(0, r)$, the functions $\beta_{m d}(G, \rho), b_{m d}(G, \rho)$ will be defined as in Definition 2.2, using again the polynomials $P_{m}$ of Lemma 2.1.

As in the real case, the indicated suprema are monotonically increasing with $\rho$, and their finiteness at $\rho_{1}$ ensures finiteness for all $\rho \leq \rho_{1}$. If $b_{m}(G, \rho)<+\infty$, at least for $\rho$ in a right neighborhood of zero, we put $b_{m}(G, 0):=\lim _{\rho \rightarrow 0^{+}} b_{m}(G, \rho)$. We also intend $\beta_{m d}(G, 0):=$ $\lim _{\rho \rightarrow 0^{+}} \beta_{m d}(G, \rho), b_{m d}(G, 0):=\lim _{\rho \rightarrow 0^{+}} b_{m d}(G, \rho)$, if these quantities are finite for $\rho$ close to zero.

Definition 2.6. Let $n \in \mathbf{N}$. A map $G$ as above is said to have the $\Pi^{n}$ property if:

(i) $G$ is $C^{n}$;

(ii) $b_{m}(G, \rho)<+\infty$ for $0 \leq m \leq n$ and all $\rho \in(0, r)$; $\sigma_{h k \ell}(G, \rho)<+\infty$ for $h, k \geq 0,1 \leq h+k \leq n, 0 \leq \ell \leq n-h-k$ and all $\rho \in[0, r)$;

(iii) the functions $b_{m}(G,),. \sigma_{h k \ell}(G,):. B_{\mathbf{C}}(0, r) \rightarrow[0,+\infty)$ are continuous for all $m, h, k, \ell$ as in ii).

The main result on complex Nemytskij operators is the following.

Proposition 2.7. Let $n, a \in N$ and $a>d / 2$. Consider functions $G: B_{\boldsymbol{C}}(0, r) \times \boldsymbol{R}^{d} \rightarrow \boldsymbol{C}, f: \boldsymbol{R}^{d} \rightarrow \boldsymbol{C}$ with the $\boldsymbol{C}$-analogues of properties (i)-(ii) in Proposition 2.4. Then $G(f, \mathrm{x}) \in H^{n}\left(\boldsymbol{R}^{d}, \boldsymbol{C}\right)$ and Eqs. (2.31-2.34) hold again, with the definitions given therein for $\gamma_{n d}(G, \rho)$ and $c_{n d}(G, \rho)$.

The case of an $\boldsymbol{x}$-independent $\boldsymbol{G}$. Let us consider a function

$$
G:(-r, r) \rightarrow \mathbf{R}, u \mapsto G(u) \quad \text { or } \quad G: B_{\mathbf{C}}(0, r) \rightarrow \mathbf{C}, z \mapsto G(z)
$$

(derivable in the ordinary sense, as many times as required in the sequel); we regard $G$ as an $x$-independent function of $(u, x)$ or $(z, x)$. Some of the previous formulae become simpler due to the vanishing of all derivatives $\nabla^{m} G(m \geq 1)$ with respect to the $x$ variables. More precisely,

$$
b_{m}(G, \rho)=0 \quad \text { for } m \geq 1, \quad \sigma_{j \ell}(G, \rho)=0 \quad \text { for } \ell \geq 1,
$$

also implying

$$
b_{m d}(G, \rho)=0 \quad \text { for } m \geq 1
$$




$$
\begin{gathered}
\beta_{m d}(G, \rho)=P_{m}\left(\nu_{j 0}=U_{m j d} \frac{\sigma_{j 0}(G, \rho)}{\rho} ; \nu_{j \ell}=0 \text { for } \ell \neq 0, \rho\right) ; \\
c_{n d}(G, \rho)=0 \quad \text { for all } n \geq 0 .
\end{gathered}
$$

$G$ has the $\Pi^{n}$ property for $n \geq 1$ if and only if it is $C^{n}$; the $\Pi^{0}$ property is possessed by $G$ if and only if $G$ is $C^{0}$ and $b_{0}(G, \rho)$ is finite and continuous in $\rho$.

Both in the real and in the complex case, we can study the Nemytskij operator sending $f \in H^{n}\left(\mathbf{R}^{d}\right) \cap H_{r}^{a}\left(\mathbf{R}^{d}\right)$ into $G(f)$, with the aid of Propositions 2.4 or 2.7. Of course, the constant function $x \in \mathbf{R}^{d} \mapsto G(0)$ is in $H^{n}\left(\mathbf{R}^{d}\right)$ for any $n$ if and only if

$$
G(0)=0 .
$$

If this happens and $G$ has the $\Pi^{n}$ property, Eq. (2.31) holds, taking in this case the form

$$
\|G(f)\|_{n} \leq \gamma_{n d}\left(G, S_{a d}\|f\|_{a}\right)\|f\|_{n} ;
$$

as in Eq. (2.32), it is $\gamma_{n d}(G, \rho):=\max \left\{b_{0}(G, \rho), \beta_{m d}(G, \rho)(m=1, \ldots, n)\right\}$. We shall exemplify this scheme in two cases: in the first one $G$ is a monomial in $z$ and $\bar{z}$, in the second one it is the hyperbolic sine. (The interest of the second example is that we see the whole machinery at work on a function with non polynomial growth).

Examples. (i) Let us put

$$
G: \mathbf{C} \rightarrow \mathbf{C}, \quad z \mapsto G(z):=z^{\mathcal{H}} \bar{z}^{\mathcal{K}},
$$

where $\mathcal{H}, \mathcal{K} \in \mathbf{N}$ and $\mathcal{H}+\mathcal{K} \neq 0$. Of course $\partial^{h} \bar{\partial}^{k} G(z)=\mathcal{H} ! \mathcal{K} ! /(\mathcal{H}-h) !(\mathcal{K}-$ $k) ! z^{\mathcal{H}-h} \bar{z}^{\mathcal{K}-k}$ for $0 \leq h \leq \mathcal{H}$ and $0 \leq k \leq \mathcal{K}$; all the other derivatives of $G$ vanish. The nonzero functions in Eqs. (2.38)-(2.39) are

$$
b_{0}(G, \rho)=\rho^{\mathcal{H}+\mathcal{K}-1},
$$

$$
\sigma_{h k 0}(G, \rho)=\frac{\mathcal{H} ! \mathcal{K} !}{(\mathcal{H}-h) !(\mathcal{K}-k) !} \rho^{\mathcal{H}+\mathcal{K}-h-k} \quad(0 \leq h \leq \mathcal{H} ; 0 \leq k \leq \mathcal{K}) ;
$$

from here, one also infers that the only nonzero functions in (2.40) are the $\sigma_{j 0}$ for $1 \leq j \leq \mathcal{H}+\mathcal{K}$, given by 


$$
\begin{aligned}
\sigma_{j 0}(G, \rho) & =\left(\sum_{h=0}^{j}\left(\begin{array}{c}
j \\
h
\end{array}\right) \frac{\mathcal{H} ! \mathcal{K} !}{(\mathcal{H}-h) !(\mathcal{K}-j+h) !}\right) \rho^{\mathcal{H}+\mathcal{K}-j} \\
& =j !\left(\sum_{h=0}^{j}\left(\begin{array}{c}
\mathcal{H} \\
h
\end{array}\right)\left(\begin{array}{c}
\mathcal{K} \\
j-h
\end{array}\right)\right) \rho^{\mathcal{H}+\mathcal{K}-j} \\
& =j !\left(\begin{array}{c}
\mathcal{H}+\mathcal{K} \\
j
\end{array}\right) \rho^{\mathcal{H}+\mathcal{K}-j} \\
& =\frac{(\mathcal{H}+\mathcal{K}) !}{(\mathcal{H}+\mathcal{K}-j) !} \rho^{\mathcal{H}+\mathcal{K}-j} .
\end{aligned}
$$

Thus, we have

$$
\begin{gathered}
\beta_{m d}(G, \rho)=P_{m}\left(\nu_{j 0}=\frac{(\mathcal{H}+\mathcal{K}) !}{(\mathcal{H}+\mathcal{K}-j) !} U_{m j d} \rho^{\mathcal{H}+\mathcal{K}-j-1} \text { for } 1 \leq j \leq \mathcal{H}+\mathcal{K} ;\right. \\
\left.\nu_{j \ell}=0 \text { otherwise }, \rho\right) .
\end{gathered}
$$

On the other hand, each coefficient $\nu_{j 0}$ in the polynomial $P_{m}$ multiplies $\rho^{j}$ (see Eq. (2.18)). From here, one easily infers

$$
\beta_{m d}(G, \rho)=B_{m d}^{\mathcal{H}+\mathcal{K}} \rho^{\mathcal{H}+\mathcal{K}-1} \quad(m \geq 1)
$$

where the $B_{m d}^{\mathcal{J}}$ are positive coefficients defined for any integer $\mathcal{J} \geq 1$, setting (2.53)

$B_{m d}^{\mathcal{J}}:=P_{m}\left(\nu_{j 0}=\frac{\mathfrak{J} !}{(\mathcal{J}-j) !} U_{m j d}\right.$ for $1 \leq j \leq \mathcal{J} ; \nu_{j \ell}=0$ otherwise,$\left.\rho=1\right)$;

this also implies

$$
\begin{gathered}
\gamma_{n d}(G, \rho)=\Gamma_{n d}^{\mathcal{H}+\mathcal{K}} \rho^{\mathcal{H}+\mathcal{K}-1} \\
\Gamma_{n d}^{\mathcal{J}}:=\max \left(1, B_{m d}^{\mathcal{J}}(m=1, \ldots, n)\right) \quad(n \in \mathbf{N}) .
\end{gathered}
$$

The final result on the Nemytskij operator $G(f)=f^{\mathcal{H}} \bar{f}^{\mathcal{X}}$ is the following, for all $n, a \in \mathbf{N}$ with $a>d / 2$ : for each $f \in H^{n}\left(\mathbf{R}^{d}, \mathbf{C}\right) \cap H^{a}\left(\mathbf{R}^{d}, \mathbf{C}\right)$ it is $f^{\mathcal{H}} \bar{f}^{\mathcal{K}} \in H^{n}\left(\mathbf{R}^{d}, \mathbf{C}\right)$ and

$$
\left\|f^{\mathcal{H}} \bar{f}^{\mathcal{K}}\right\|_{n} \leq \Gamma_{n d}^{\mathcal{H}+\mathcal{K}}\left(S_{a d}\|f\|_{a}\right)^{\mathcal{H}+\mathcal{K}-1}\|f\|_{n} .
$$


A slightly weaker, but simpler bound can be obtained recalling that all $U$ coefficients are bounded by 1 . For each $m \geq 1$, this implies

$$
\begin{gathered}
B_{m d}^{\mathcal{J}} \leq P_{m}\left(\nu_{j 0}=\frac{\mathfrak{J} !}{(\mathcal{J}-j) !} \text { for } 1 \leq j \leq \mathcal{J} ; \nu_{j \ell}=0 \text { otherwise }, \rho=1\right) \\
\quad=\left.\frac{d^{m}}{d \xi^{m}}\right|_{\xi=0} e^{\mathcal{J} \xi}=\mathcal{J}^{m} ;
\end{gathered}
$$

the equality at the beginning of the last line can be obtained directly from the relation (2.17) defining $P_{m}$, to be applied at $\xi=0$ to the function $F(u, \xi):=u^{\mathcal{g}}$.

From (2.54) and (2.56) one readily infers $\Gamma_{n d}^{\mathcal{J}} \leq \mathcal{g}^{n}$ for all $n \geq 0$, whence

$$
\left\|f^{\mathcal{H}} \bar{f}^{\mathcal{K}}\right\|_{n} \leq(\mathcal{H}+\mathcal{K})^{n}\left(S_{a d}\|f\|_{a}\right)^{\mathcal{H}+\mathcal{K}-1}\|f\|_{n} .
$$

Let us recall that, in the previous work [18], we have discussed some inequalities for the norm $\|f g\|_{n}$ of a product, implying directly an estimate of the form $\left\|f^{\mathcal{H}} \bar{f}^{\mathcal{K}}\right\|_{n} \leq K_{\text {nad }}\|f\|_{a}\|f\|_{n}$ for all $\mathcal{H}, \mathcal{K}$ with $\mathcal{H}+\mathcal{K}=2\left(^{\ddagger}\right)$. In all the tests we have performed, the upper bounds of [18] on the constants $K_{\text {nad }}$ and the bounds $\Gamma_{n d}^{\mathcal{H}+\mathcal{K}} S_{a d}$ arising from (2.55), with $\mathcal{H}+\mathcal{K}=2$, are very close numerically. A result of [18] also implies the weaker bound (2.57) for $\mathcal{H}+\mathcal{K}=2$.

For $\mathcal{H}+\mathcal{K}>2$, an estimation of $\left\|f^{\mathcal{H}} \bar{f}^{\mathcal{X}}\right\|_{n}$ could be performed by iteration of the bounds [18] on the product of two functions; however, the estimates derived in this elementary way would be sensibly rougher than (2.55) or (2.57).

(ii) We consider the hyperbolic function

$$
G: \mathbf{R} \rightarrow \mathbf{R}, \quad u \mapsto G(u):=\sinh u .
$$

From Eqs. (2.27) (2.28) we get

$$
b_{0}(\sinh , \rho)=\frac{\sinh \rho}{\rho}, \quad b_{m}(\sinh , \rho)=0 \quad(m \geq 1)
$$

$$
\begin{gathered}
\sigma_{j 0}(\sinh , \rho)=\sinh \rho \quad(j \text { even }), \quad \sigma_{j 0}(\sinh , \rho)=\cosh \rho \quad(j \text { odd }), \\
\sigma_{j \ell}(\sinh , \rho)=0 \quad \text { for } \ell \neq 0 .
\end{gathered}
$$

${ }^{\ddagger}$ In [18], it was assumed either $0 \leq n \leq d / 2<a$ or $d / 2<a \leq n$. 
From these objects and Eq. (2.29) one computes

$$
\begin{gathered}
\beta_{m d}(\sinh , \rho)=P_{m}\left(\nu_{j 0}=U_{m j d} \frac{\sinh \rho}{\rho} \text { for } j \text { even } ;\right. \\
\left.\nu_{j 0}=U_{m j d} \frac{\cosh \rho}{\rho} \text { for } j \text { odd } ; \nu_{j \ell}=0 \text { for } \ell \neq 0, \rho\right)
\end{gathered}
$$

and $\gamma_{n d}(\sinh , \rho)=\max \left(b_{0}(\sinh , \rho), \beta_{1 d}(\sinh , \rho), \ldots, \beta_{n d}(\sinh , \rho)\right)$. We have

$$
\|\sinh f\|_{n} \leq \gamma_{n d}\left(\sinh , S_{a d}\|f\|_{a}\right)\|f\|_{n}
$$

for all $n, a$ as usually and $f \in H^{n}\left(\mathbf{R}^{d}, \mathbf{R}\right) \cap H^{a}\left(\mathbf{R}^{d}, \mathbf{R}\right)$. For instance, let us choose $n=2$; this requires use of the polynomials $P_{1}, P_{2}$ in the Table, from which one computes $\beta_{1 d}(\sinh , \rho)=U_{11 d} \cosh \rho$ and $\beta_{2 d}(\sinh , \rho)=$ $U_{22 d} \rho \sinh \rho+U_{21 d} \cosh \rho$. Eq. (2.62) holds with

$$
\gamma_{2 d}(\sinh , \rho)=\max \left(\frac{\sinh \rho}{\rho}, U_{11 d} \cosh \rho, U_{22 d} \rho \sinh \rho+U_{21 d} \cosh \rho\right) .
$$

\section{More on tensor calculus. Iterated derivatives of Nemytskij operators}

The purpose of this Section and of the next two is to give some tools for proving the main results of the paper. Here we concentrate on some algebraic aspects of tensor calculus, on the differentiation of tensors and on a general formula for the derivatives of any order of a composite function $G(f, \mathrm{x})$. In Section 4 we will connect these results with the polynomials $P_{m}$; in Section 5 we will be concerned with functional spaces of tensor-valued mappings, and some related inequalities.

We already introduced the tensor powers $\otimes^{\ell} \mathbf{C}^{d}$. For $\ell, m \in \mathbf{N}$, the tensor product operation

$$
\otimes:\left(\otimes^{\ell} \mathbf{C}^{d}\right) \times\left(\otimes^{m} \mathbf{C}^{d}\right) \rightarrow \otimes^{\ell+m} \mathbf{C}^{d}, \quad(T, U) \mapsto T \otimes U
$$

is defined setting

$$
(T \otimes U)_{\lambda_{1} \ldots \lambda_{\ell} \mu_{1} \ldots \mu_{m}}:=T_{\lambda_{1} \ldots \lambda_{\ell}} U_{\mu_{1} \ldots \mu_{m}}
$$

for $\lambda_{1}, \ldots, \mu_{m} \in\{1, \ldots, d\}$ (for $\ell=0, T \otimes U$ means the product $T U$ between the complex number $T$ and $U$, and the same is understood for $m=0$ ). The product $\otimes$ is clearly associative. 
Let $\ell \in \mathbf{N}_{0}$ and $\sigma$ a permutation of $\{1, \ldots, \ell\}$; then, we a have a (linear) permutation operator

$$
\mathcal{P}_{\sigma}: \otimes^{\ell} \mathbf{C}^{d} \rightarrow \otimes^{\ell} \mathbf{C}^{d}, \quad\left(\mathcal{P}_{\sigma} T\right)_{\lambda_{1} \ldots \lambda_{\ell}}:=T_{\lambda_{\sigma(1)} \ldots \lambda_{\sigma(\ell)}} .
$$

A tensor $T \in \otimes^{\ell} \mathbf{C}^{d}$ is said to be symmetric if $\mathcal{P}_{\sigma} T:=T$ for all permutations $\sigma$; any $T \in \otimes{ }^{0} \mathbf{C}^{d}=\mathbf{C}$ is symmetric by definition. Let $\ell \in \mathbf{N}$; we put

$$
\vee^{\ell} \mathbf{C}^{d}:=\left\{T \in \otimes^{\ell} \mathbf{C}^{d} \mid T \text { is symmetric }\right\}
$$

and introduce a symmetrisation operator

$$
\mathcal{S}: \otimes^{\ell} \mathbf{C}^{d} \rightarrow \vee^{\ell} \mathbf{C}^{d}, \quad \mathcal{S}:=\frac{1}{\ell !} \sum_{\sigma \in \ell !} \mathcal{P}_{\sigma}
$$

where $\ell$ ! stands for the set of all permutations of $\{1, \ldots, \ell\}$ (whose cardinality is $\ell$ !). Note that $\mathcal{S}$ is the identity map for $\ell=1$; we intend $\mathcal{S}$ to be the identity for $\ell=0$ also. A tensor $T$ is symmetric if and only if $\mathcal{S} T=T$.

For $\ell, m \in \mathbf{N}$, the symmetrised tensor product operation $\vee$ is defined setting

$$
\vee:\left(\otimes^{\ell} \mathbf{C}^{d}\right) \times\left(\otimes^{m} \mathbf{C}^{d}\right) \rightarrow \vee^{\ell+m} \mathbf{C}^{d}, \quad(T, U) \mapsto T \vee U:=\mathcal{S}(T \otimes U) .
$$

This operation is commutative and associative (see the Appendix) :

$$
U \vee T=T \vee U, \quad(T \vee U) \vee V=T \vee(U \vee V)
$$

for arbitrary tensors $T, U$ as above and $V \in \otimes^{p} \mathbf{C}^{d}$. (Often, $\vee$ is considered to act on symmetric tensors only; this leads to the so-called symmetric tensor algebra, see, e.g., [20], [11]). If $T \in \otimes^{\ell} \mathbf{C}^{d}$ and $q \in \mathbf{N}$, we will write

$$
\vee^{q} T:=T \vee T \ldots \vee T \quad(q \text { times }),
$$

intending this to be 1 if $q=0$.

We already defined the norm || of tensors, see Eq. (2.2); for all $T, U$ it is

$$
|T \otimes U|=|T||U|, \quad|T \vee U| \leq|T||U|
$$

(see the Appendix). Sometimes, we need to employ the complex conjugate $\bar{T}$ of a tensor $T \in \otimes^{\ell} \mathbf{C}^{d}$; this has components

$$
\bar{T}_{\lambda_{1} \ldots \lambda_{\ell}}:=\overline{T_{\lambda_{1} \ldots \lambda_{\ell}}} .
$$


We now pass to spaces of tensor-valued functions, or tensor fields

$$
T: \mathbf{R}^{d} \mapsto \otimes^{\ell} \mathbf{C}^{d} \text { or } \vee^{\ell} \mathbf{C}^{d}, \quad x=\left(x_{1}, \ldots, x_{d}\right) \mapsto T(x) ;
$$

the products $\otimes, \vee$, the conjugate - , etc. are defined pointwisely on such functions. $C^{n}\left(\mathbf{R}^{d}, \otimes^{\ell} \mathbf{C}^{d}\right.$ or $\left.\vee^{\ell} \mathbf{C}^{d}\right)$ is the space of mappings as in (3.11), where all components are $C^{n}$ in the usual sense.

Given a tensor field $T$ as above, of class $C^{1}$, we define the derivative $\nabla T$ to be the tensor field of order $\ell+1$ with components

$$
(\nabla T)_{\lambda_{1} \ldots \lambda_{\ell+1}}:=\partial_{\lambda_{\ell+1}} T_{\lambda_{1} \ldots \lambda_{\ell}} .
$$

For $p \in \mathbf{N}$ we denote with $\nabla^{p}$ the $p$-th power of the operator $\nabla$ (acting on $C^{p}$ tensor fields); for any $T \in C^{p}\left(\mathbf{R}^{d}, \otimes^{\ell} \mathbf{C}^{d}\right)$, the tensor field $\nabla^{p} T$ $\in C\left(\mathbf{R}^{d}, \otimes^{\ell+p} \mathbf{C}^{d}\right)$ has components

$$
\left(\nabla^{p} T\right)_{\lambda_{1} \ldots \lambda_{\ell+p}}=\left(\partial_{\lambda_{\ell+p}} \ldots \partial_{\lambda_{\ell+1}}\right) T_{\lambda_{1} \ldots \lambda_{\ell}}
$$

(of course derivatives $\partial_{\lambda_{\ell+p}}, \ldots \partial_{\lambda_{\ell+1}}$ commute, so their ordering is immaterial). In the sequel, we often employ the symmetrised derivative

$$
\nabla_{\mathcal{S}} T:=\mathcal{S}(\nabla T)
$$

and denote with $\nabla_{\mathcal{S}}^{p}$ the $p$-th power of the operator $\nabla_{\mathcal{S}}$. On any function $f \in C^{p}\left(\mathbf{R}^{d}, \mathbf{C}\right)$, we have

$$
\nabla_{\mathcal{S}}^{p} f=\nabla^{p} f=\text { the already defined tensor field (2.6) . }
$$

One can write a Leibnitz rule for $\nabla$ and the product $\otimes$, see the Appendix; we will not use this rule directly and always refer to a symmetrized version, also proved in the Appendix. The "symmetrized" Leibnitz rule reads

$$
\nabla_{\mathcal{S}}(T \vee U)=\left(\nabla_{\mathcal{S}} T\right) \vee U+T \vee\left(\nabla_{\mathcal{S}} U\right)
$$

for all $C^{1}$ tensor fields $T: \mathbf{R}^{d} \rightarrow \otimes^{\ell} \mathbf{C}^{d}$ and $U: \mathbf{R}^{d} \rightarrow \otimes^{m} \mathbf{C}^{d}$.

What has been said up to now for complex tensors can be rephrased in the real case; so, we can define the product $\otimes$ between tensors $T \in \otimes^{\ell} \mathbf{R}^{d}$, $U \in \otimes \otimes^{m} \mathbf{R}^{d}$, the symmetrisation operator $\mathcal{S}$, the $\vee$ product and the derivatives $\nabla, \nabla_{\mathcal{S}}$ of real tensor fields, which satisfy again the previously mentioned Leibnitz rules.

Parameter dependent tensor fields. We begin from the real case. We consider tensor fields depending on a supplementary real variable $u$, 
i.e., mappings

$$
T:(-r, r) \times \mathbf{R}^{d} \rightarrow \otimes^{\ell} \mathbf{R}^{d}, \quad(u, x) \mapsto T(u, x) ;
$$

we define all previous operations $\otimes, \mathcal{S}, \vee, \nabla, \nabla_{\mathcal{S}}$ treating $u$ as a parameter, e.g., $(T \otimes U)(u, x):=T(u, x) \otimes U(u, x),(\nabla T)_{\lambda_{1} \ldots \lambda_{\ell+1}}(u, x):=$ $\partial_{\lambda_{\ell+1}} T_{\lambda_{1} \ldots \lambda_{\ell}}(u, x)$ with $\partial_{\lambda_{i}}:=\partial / \partial x_{\lambda_{i}}$. As in Section 2, we denote with $\partial$ the derivative $\partial / \partial u$. For any $u$-dependent tensor field $T$ of order $\ell$ as in (3.17) and class $C^{1}, \partial T$ is the $u$-dependent tensor field, again of order $\ell$, with components

$$
(\partial T)_{\lambda_{1} \ldots \lambda_{\ell}}:=\partial\left(T_{\lambda_{1} \ldots \lambda_{\ell}}\right) .
$$

Of course $\partial$ commutes with $\nabla$ and $\nabla_{\mathcal{S}}$. We can iterate the operations $\partial, \nabla, \nabla_{\mathcal{S}}$; so, for $T$ of order $\ell$ and class $C^{j+m}$, the tensor fields $\partial^{j} \nabla^{m} T$, $\partial^{j} \nabla_{\mathcal{S}}^{m} T$ (both of order $\ell+m$ ) are defined.

The case of complex tensor fields, depending a complex parameter $z$, is worked out similarly. In this case we consider mappings

$$
T: B_{\mathbf{C}}(0, r) \times \mathbf{R}^{d} \rightarrow \otimes^{\ell} \mathbf{C}^{d}, \quad(z, x) \mapsto T(z, x) ;
$$

any such map is said to be $C^{n}$ if its derivatives w.r.t. $z, \bar{z}$ and the real variables $x$, up to total order $n$, exist everywhere and are continuous. The $z$-dependent tensor fields are acted upon by the derivatives $\partial:=\partial / \partial z$, $\bar{\partial}:=\partial / \partial \bar{z}, \nabla, \nabla_{\mathcal{S}}$ and their iterates.

Iterated derivatives of composite functions. Again, we start from the real case. We consider a $u$-dependent tensor field of arbitrary order $\ell$

$$
H:(-r, r) \times \mathbf{R}^{d} \rightarrow \otimes{ }^{\ell} \mathbf{R}^{d}, \quad(u, x) \mapsto H(u, x) .
$$

We associate to $H$ a "tensor" Nemytskij operator sending a function $f: \mathbf{R}^{d} \mapsto(-r, r)$ into the tensor field

$$
H(f, \mathrm{x}): \mathbf{R}^{d} \rightarrow \otimes^{\ell} \mathbf{R}^{d}, \quad x \mapsto H(f(x), x) .
$$

If $H$ is a tensor field of order 0, i.e., a real valued function $G$, we recover definition (1.1) of $G(f, \mathrm{x})$ and the usual notion of Nemytskij operator. For $\ell$ arbitrary, we are interested in derivatives of $H(f, \mathrm{x})$. Assume $H, f$ to be $C^{1}$; then

$$
\begin{aligned}
\nabla(H(f, \mathrm{x}))_{\lambda_{1} \ldots \lambda_{\ell+1}} & =\partial_{\lambda_{\ell+1}}\left(H_{\lambda_{1} \ldots \lambda_{\ell}}(f, \mathrm{x})\right) \\
& =\left(\partial H_{\lambda_{1} \ldots \lambda_{\ell}}\right)(f, \mathrm{x}) \partial_{\lambda_{\ell+1}} f+\left(\partial_{\lambda_{\ell+1}} H_{\lambda_{1} \ldots \lambda_{\ell}}\right)(f, \mathrm{x})
\end{aligned}
$$


(recall $\partial:=\partial / \partial u)$. In compact form, this amounts to write

$$
\nabla(H(f, \mathrm{x}))=\partial H(f, \mathrm{x}) \otimes \nabla f+\nabla H(f, \mathrm{x}) ;
$$

we can symmetrise this result by application of $\mathcal{S}$ to both sides, the result being

$$
\nabla_{\mathcal{S}}(H(f, \mathrm{x}))=\partial H(f, \mathrm{x}) \vee \nabla f+\nabla_{\mathcal{S}} H(f, \mathrm{x}) .
$$

Now, we consider the zero order case $H=G:(-r, r) \times \mathbf{R}^{d} \rightarrow \mathbf{R}$. We are interested in the iterates $\nabla^{m}(G(f, \mathbf{x}))\left(m \in \mathbf{N}_{0}\right)$. These are computed using as basic tools: the equality $\nabla^{m}=\nabla_{\mathcal{S}}^{m}$ on real-valued functions, Eq. (3.23), the Leibnitz rule (3.16), and the fact that $V$ is just the ordinary product when one of the two factors is of order zero. For example,

$$
\begin{aligned}
\nabla(G(f, \mathrm{x}))= & \nabla_{\mathcal{S}}(G(f, \mathrm{x}))=\partial G(f, \mathrm{x}) \nabla f+\nabla G(f, \mathrm{x}) \\
\nabla^{2}(G(f, \mathrm{x}))= & \nabla_{\mathcal{S}}(\partial G(f, \mathrm{x}) \nabla f+\nabla G(f, \mathrm{x})) \\
= & \partial^{2} G(f, \mathrm{x}) \nabla f \vee \nabla f+2 \partial \nabla G(f, \mathrm{x}) \vee \nabla f \\
& +\partial G(f, \mathrm{x}) \nabla^{2} f+\nabla^{2} G(f, \mathrm{x}) .
\end{aligned}
$$

To generalize this to any order, we need some notations. Let us put

$$
\begin{gathered}
\mathbf{N}^{(\infty)}:=\left\{p=\left(p_{1}, p_{2}, \ldots\right) \mid p_{s} \in \mathbf{N} \text { for all } s \in \mathbf{N}_{0},\right. \\
\left.p_{s} \neq 0 \text { for finitely many } s\right\} \\
D_{m}:=\left\{p \in \mathbf{N}^{(\infty)} \mid 1 \leq p_{1}+2 p_{2}+3 p_{3}+\ldots \leq m\right\}
\end{gathered}
$$

for all integers $m, j, w$ such that $m \geq 1,1 \leq j \leq w$. The set $D_{j w}$ is non empty for any pair $(j, w)$ as before $\left({ }^{\S}\right)$, and $D_{m}=\cup_{1 \leq w \leq m} D_{j w}$ $=\cup_{1 \leq j \leq m} D_{j, m-\ell}$. Let us also recall that $\vee^{q}$ means the $q$-th power w.r.t $0 \leq \ell \leq m-j$ the $\vee$ product, see Eq. (3.8).

Proposition 3.1. Let $m \in \boldsymbol{N}_{0}$; then, there exists a unique family of positive, integer coefficients

$$
\left(P_{m \mid p}\right)_{p \in D_{m}}
$$

\footnotetext{
§In fact, let $\sigma, \rho$ be the integers such that $\sigma \geq 1,0 \leq \rho \leq j-1$ and $w=j \sigma+\rho$; then, $D_{j w}$ contains at least the sequence $p_{s}:=(j-\rho) \delta_{s \sigma}+\rho \delta_{s, \sigma+1}$.
} 
such that for each dimension $d$, each $C^{m}$ function $G:(-r, r) \times \boldsymbol{R}^{d} \rightarrow \boldsymbol{R}$, $(u, x) \mapsto G(u, x)$ and each $C^{m}$ function $f: \boldsymbol{R}^{d} \rightarrow(-r, r)$ it is

$$
\begin{gathered}
\nabla^{m}(G(f, \mathrm{x}))=\sum_{\substack{1 \leq j \leq m \\
0 \leq \ell \leq m-j}} \partial^{j} \nabla^{\ell} G(f, \mathrm{x}) \vee \\
\vee \sum_{p \in D_{j, m-\ell}} P_{m \mid p}\left(\vee^{p_{1}} \nabla^{1} f\right) \vee\left(\bigvee^{p_{2}} \nabla^{2} f\right) \ldots+\nabla^{m} G(f, \mathrm{x}) .
\end{gathered}
$$

These coefficients are given by

$$
P_{m \mid p}=\frac{m !}{\left(m-p_{1}-2 p_{2}-\ldots\right) !} \frac{1}{(1 !)^{p_{1}} p_{1} !(2 !)^{p_{2}}\left(p_{2}\right) ! \ldots}
$$

for all $p \in D_{m}$.

Remarks. (i) For $d=1$ and an $x$-independent $G$, this statement is known as the Faà di Bruno formula, see e.g. [5]; we acknowledge S. Paveri Fontana for bibliographical indications concerning this case. Of course, for $d=1$ the $\vee$ product collapses into the usual product of real numbers. Our formulation in terms of tensor products holds for an arbitrary space dimension $d$ (and $x$-dependent $G$ ).

Some multidimensional extensions of the Faà di Bruno formula, not using the tensor language, were proposed in the literature (see, e.g., [8], [15]); another one was suggested to us by G. Meloni [14], to whom we are grateful for general discussions on this subject and its relations with the Stirling numbers.

(ii) Eq. (3.30) can be written in terms of the Bell polynomials and some differential operators. The Bell polynomials $Y_{w}\left(t_{1}, t_{2}, \ldots, t_{w}\right)$ [5] (which are very similar to the Schur polynomials [10]) are defined by the formal expansion $\exp \left(\sum_{r=1}^{\infty} t_{r} \alpha^{r} / r !\right)=1+\sum_{w=1}^{\infty} Y_{w}\left(t_{1}, t_{2}, \ldots, t_{w}\right) \alpha^{w} / w$ !, and given explicitly by

$$
Y_{w}\left(t_{1}, t_{2}, \ldots, t_{w}\right)=\sum_{\substack{p \in \mathbf{N}^{(\infty)}, p_{1}+2 p_{2}+s_{3}+\ldots=w}} \frac{w !}{(1 !)^{p_{1}} p_{1} !(2 !)^{p_{2}} p_{2} !(3 !)^{p_{3}} p_{3} ! \ldots} t^{p_{1}} t_{2}{ }^{p_{2}} t_{3}{ }^{p_{3}} \ldots
$$

for each integer $w \geq 1$. Comparing with Eqs. (3.30) (3.31), one finds that the formula for the derivatives of a composite function can be written in 
the symbolic form

$$
\begin{aligned}
\nabla^{m}(G(f, \mathrm{x}))= & \left(\sum_{0 \leq \ell \leq m-1}\left(\begin{array}{c}
m \\
\ell
\end{array}\right) Y_{m-\ell}\left(\nabla^{1} f \vee \partial, \nabla^{2} f \vee \partial, \ldots\right) \nabla^{\ell}\right) \\
& \times(G)(f, \mathrm{x})+\nabla^{m} G(f, \mathrm{x}),
\end{aligned}
$$

the sum denoting an operator which acts on the function $(u, x) \mapsto G(u, x)$.

Proof of Proposition 3.1. First of all, one can prove by recursion over $m$ the existence of a family of nonnegative, integer coefficients $\left(P_{m \mid p}\right)$ fulfilling (3.30) for any $d, G$ and $f$. The existence of this family for $m=1$ (and $m=2$ ) is known from (3.24) (and (3.25)). Assuming existence for some $m$, one can derive existence at order $m+1$ computing $\nabla^{m+1}(G(f, \mathrm{x}))=\nabla_{S}\left(\nabla^{m}(G(f, \mathrm{x}))\right)$ from Eq. (3.30). Differentiating this equation via the Leibnitz rule (3.16) and Eq. (3.23), one obtains a sum of terms with nonnegative, integer coefficients, each one of a type included in the $(m+1)$-th version of Eq. (3.30).

Let us pass to prove uniqueness of the coefficients, and derive the explicit expression (3.31); this will also make clear that $P_{m \mid p} \neq 0$ for all $p$. Let us consider any family $\left(P_{m \mid p}\right)$ fulfilling (3.30) for a given $m$ and all $d, G, f$. We apply Eq. (3.30) with this set of coefficients and $d=1$, calling $\xi$ the $x$ variable; the operator $\nabla$ is just the ordinary derivative $d / d \xi$, and (3.30) becomes (adding primes to indices $j, \ell$, for future conveniency)

$$
\begin{aligned}
\frac{d^{m}}{d \xi^{m}}(G(f(\xi), \xi))= & \sum_{\substack{1 \leq j^{\prime} \leq m \\
0 \leq \ell^{\prime} \leq m-j^{\prime}}} \frac{\partial^{j^{\prime}+\ell^{\prime}} G}{\partial u^{j^{\prime}} \partial \xi^{\ell^{\prime}}}(f(\xi), \xi) \sum_{p \in D_{j^{\prime}, m-\ell^{\prime}}} P_{m \mid p}\left(\frac{d f(\xi)}{d \xi}\right)^{p_{1}} \\
& \times\left(\frac{d^{2} f(\xi)}{d \xi^{2}}\right)^{p_{2}} \ldots+\frac{\partial^{m} G}{\partial \xi^{m}}(f(\xi), \xi)
\end{aligned}
$$

for all $C^{m}$ functions $G:(-r, r) \times \mathbf{R} \rightarrow \mathbf{R}$ and $f: \mathbf{R} \rightarrow(-r, r)$.

Now, we choose a sequence $p=\left(p_{1}, p_{2}, \ldots\right)$ in the domain of the family (3.29), and set

$$
\begin{aligned}
& j:=p_{1}+p_{2}+\ldots, \quad \ell:=m-p_{1}-2 p_{2}-\ldots \\
& G(u, \xi):=\frac{u^{j} \xi^{\ell}}{j ! \ell !}, \quad f(\xi):=a_{1} \xi+a_{2} \frac{\xi^{2}}{2 !}+a_{3} \frac{\xi^{3}}{3 !}+\ldots
\end{aligned}
$$

where $a_{s}$ is an arbitrary real parameter if $p_{s} \neq 0$, and $a_{s}:=0$ if $p_{s}=0$. Let us evaluate at $\xi=0$ all derivatives in (3.34). The only non vanishing 
derivatives $\left(\partial^{j^{\prime}+\ell^{\prime}} G / \partial u^{j^{\prime}} \partial \xi^{\ell^{\prime}}\right)$ at $(f(0), 0)=(0,0)$ occur for $j^{\prime}=j, \ell^{\prime}=\ell$ and equal 1; furthermore $\left(d^{s} f / d \xi^{s}\right)(0)=a_{s}$. Thus, inserting these choices of $G, f$ into Eq. (3.34) and setting $\xi=0$, we obtain

$$
\left.\frac{1}{j ! \ell !} \frac{d^{m}}{d \xi^{m}}\right|_{\xi=0}\left(a_{1} \xi+a_{2} \frac{\xi^{2}}{2 !}+\ldots\right)^{j} \xi^{\ell}=P_{m \mid p} a_{1}{ }^{p_{1}} a_{2}{ }^{p_{2}} \ldots
$$

intending $0^{0}:=1$; this implies

$$
P_{m \mid p}=\frac{m !}{j ! \ell !} \times \text { coefficient of } a_{1}^{p_{1}} a_{2}^{p_{2}} \ldots \xi^{m} \text { in }\left(\sum_{s} a_{s} \frac{\xi^{s}}{s !}\right)^{j} \xi^{\ell} .
$$

Computing the indicated coefficient in the above polynomial, one obtains for $P_{m \mid p}$ the expression (3.31); the calculation is based on the identity

$$
\left(z_{1}+z_{2}+z_{3} \ldots\right)^{j}=\sum_{\substack{c_{1}, c_{2}, \ldots \in \mathbf{N} \\ c_{1}+c_{2}+\ldots=j}} \frac{j !}{c_{1} ! c_{2} ! \ldots} z_{1}^{c_{1}} z_{2}{ }^{c_{2}} \ldots,
$$

to be applied with $z_{s}:=a_{s} \xi^{s} / s !$.

We pass to the complex case, and consider a $z$-dependent tensor field

$$
H: B_{\mathbf{C}}(0, r) \times \mathbf{R}^{d} \rightarrow \otimes^{\ell} \mathbf{C}^{d}, \quad(z, x) \mapsto H(z, x) ;
$$

then a tensor Nemytskij operator is defined, sending a function $f: \mathbf{R}^{d} \mapsto$ $B_{\mathbf{C}}(0, r)$ into the tensor field

$$
H(f, \mathrm{x}): \mathbf{R}^{d} \rightarrow \otimes^{\ell} \mathbf{C}^{d}, \quad x \mapsto H(f(x), x) .
$$

Let us discuss the derivatives of $H(f, \mathrm{x})$ : it is readily shown that, for $H$ and $f$ of class $C^{1}$,

$$
\nabla(H(f, \mathrm{x}))=\partial H(f, \mathrm{x}) \otimes \nabla f+\bar{\partial} H(f, \mathrm{x}) \otimes \overline{\nabla f}+\nabla H(f, \mathrm{x}),
$$

with $\overline{\nabla f}$ denoting the complex conjugate of $\nabla f$ (recall Eq. (3.10)). The symmetrised version of this identity is

$$
\nabla_{\mathcal{S}}(H(f, \mathrm{x}))=\partial H(f, \mathrm{x}) \vee \nabla f+\bar{\partial} H(f, \mathrm{x}) \vee \overline{\nabla f}+\nabla_{\mathcal{S}} H(f, \mathrm{x}) ;
$$

this can be used, together with the Leibnitz rule (3.16) and the equality $\nabla_{\mathcal{S}}^{m}=\nabla^{m}$ on $\mathbf{C}$-valued functions, to obtain the complex analogue of Proposition 3.1. We formulate this analogue keeping the notation $\mathbf{N}^{(\infty)}$ for the set of sequences (3.26), and setting

$\mathrm{D}_{m}:=\left\{(p, q) \in \mathbf{N}^{(\infty)} \mid 1 \leq p_{1}+2 p_{2}+3 p_{3}+\ldots+q_{1}+2 q_{2}+3 q_{3}+\ldots \leq m\right\}$ 


$$
\begin{gathered}
\mathrm{D}_{h k w}:=\left\{(p, q) \in \mathbf{N}^{(\infty)} \times \mathbf{N}^{(\infty)} \mid p_{1}+p_{2}+\ldots=h, \quad q_{1}+q_{2}+\ldots=k,\right. \\
\left.p_{1}+2 p_{2}+3 p_{3}+\ldots+q_{1}+2 q_{2}+3 q_{3}+\ldots=w\right\}
\end{gathered}
$$

for all integers $m, h, k, w$ such that $m \geq 1, h, k \geq 0,1 \leq h+k \leq$ $w$. All sets $\mathrm{D}_{h k w}$ are nonempty $\left({ }^{\top}\right)$, and $\mathrm{D}_{m}=\cup_{1 \leq w \leq m, h, k \geq 0} \mathrm{D}_{h k w}=$ $\underset{\substack{h, k \geq 0,1 \leq h+k \leq m \\ 0 \leq \ell \leq m-h-k}}{\cup_{h k, m-\ell}} \mathrm{D}_{h}$

Proposition 3.2. Let $m \in \boldsymbol{N}_{0}$. Then, there exists a unique family of positive, integer coefficients

$$
\left(P_{m \mid p q}\right)_{(p, q) \in \mathrm{D}_{m}}
$$

such that for each dimension d, each $C^{m}$ function $G: B_{C}(0, r) \times \boldsymbol{R}^{d} \rightarrow \boldsymbol{C}$, $(z, x) \mapsto G(z, x)$ and each $C^{m}$ function $f: \boldsymbol{R}^{d} \rightarrow B_{C}(0, r)$ it is

$$
\begin{aligned}
& \nabla^{m}(G(f, \mathrm{x}))=\sum_{\substack{h, k \geq 0,1 \leq h+k \leq m \\
0 \leq \ell \leq m-h-k}} \partial^{h} \bar{\partial}^{k} \nabla^{\ell} G(f, \mathrm{x}) \\
& \vee \sum_{(p, q) \in \mathrm{D}_{h k, m-\ell}} P_{m \mid p q}\left(\vee^{p_{1}} \nabla^{1} f\right) \vee\left(\vee^{p_{2}} \nabla^{2} f\right) \ldots \\
& \left(\vee^{q_{1}} \overline{\nabla^{1} f}\right) \vee\left(\vee^{q_{2}} \overline{\nabla^{2} f}\right) \ldots+\nabla^{m} G(f, \mathrm{x}) .
\end{aligned}
$$

These coefficients are given by

$$
\begin{aligned}
P_{m \mid p q}= & \frac{m !}{\left(m-p_{1}-2 p_{2}-\ldots-q_{1}-2 q_{2}-\ldots\right) !} \\
& \times \frac{1}{(1 !)^{p_{1}} p_{1} !(2 !)^{p_{2}}\left(p_{2}\right) ! \ldots(1 !)^{q_{1}} q_{1} !(2 !)^{q_{2}}\left(q_{2}\right) ! \ldots}
\end{aligned}
$$

for all $(p, q) \in \mathrm{D}_{m}$.

Proof. It is similar to the proof of Proposition 3.1. The existence of a set of nonnegative, integer coefficients fulfilling (3.46) for all $d, G$ and $f$ is proved by recursion over $m$. Now, let us consider for given $m$ a family of coefficients $\left(P_{m \mid p q}\right)$ fulfilling (3.46), and prove these coefficients to be uniquely determined as in Eq. (3.47), which also implies their positiveness. To this purpose, it suffices to consider Eq. (3.46) for $d=1$, calling $\xi$ the $x$

"The pair $(h, k)$ contains at least a nonzero element, say $h$. Let $\sigma, \rho$ be the integers such that $\sigma \geq 1,0 \leq \rho \leq h-1$ and $w-k=h \sigma+\rho$; then, $D_{h k w}$ contains at least the pair $(p, q)$ such that $p_{s}:=(h-\rho) \delta_{s, \sigma}+\rho \delta_{s, \sigma+1}$ and $q_{s}:=k \delta_{s, 1}$. 
variable (and adding primes to indices), which gives

$$
\begin{aligned}
& \frac{d^{m}}{d \xi^{m}}(G(f(\xi), \xi))=\sum_{\substack{h^{\prime}, k^{\prime} \geq 0,1 \leq h^{\prime}+k^{\prime} \leq m \\
0 \leq \ell^{\prime} \leq m-h^{\prime}-k^{\prime}}} \frac{\partial^{h^{\prime}+k^{\prime}+\ell^{\prime}} G}{\partial z^{h^{\prime}} \partial \bar{z}^{k^{\prime}} \partial \xi^{\ell^{\prime}}}(f(\xi), \xi) \times \\
& \quad \times \sum_{(p, q) \in \mathrm{D}_{h^{\prime} k^{\prime}, m-\ell^{\prime}}} P_{m \mid p q}\left(\frac{d f(\xi)}{d \xi}\right)^{p_{1}}\left(\frac{d^{2} f(\xi)}{d \xi^{2}}\right)^{p_{2}} \ldots \\
& \quad \times\left(\frac{\overline{d f(\xi)}}{d \xi}\right)^{q_{1}}\left(\frac{\overline{d^{2} f(\xi)}}{d \xi^{2}}\right)^{q_{2}} \ldots+\frac{\partial^{m} G}{\partial \xi^{m}}(f(\xi), \xi)
\end{aligned}
$$

for all $C^{m}$ functions $G: B_{\mathbf{C}}(0, r) \times \mathbf{R} \rightarrow \mathbf{C}$ and $f: \mathbf{R} \rightarrow B_{\mathbf{C}}(0, r)$.

We choose a pair $(p, q)$ in the domain of the family $(3.45)$, and set

$h:=p_{1}+p_{2}+\ldots, \quad k:=q_{1}+q_{2}+\ldots, \quad \ell:=m-p_{1}-2 p_{2}-\ldots-q_{1}-2 q_{2}-\ldots$,

$$
G(z, \xi):=\frac{z^{h} \bar{z}^{k} \xi^{\ell}}{h ! k ! \ell !}, \quad f(\xi):=a_{1} \xi+a_{2} \frac{\xi^{2}}{2 !}+a_{3} \frac{\xi^{3}}{3 !}+\ldots
$$

where $a_{s}$ is an arbitrary complex parameter if $\left(p_{s}, q_{s}\right) \neq(0,0)$, and $a_{s}:=0$ if $\left(p_{s}, q_{s}\right)=(0,0)$. Inserting these choices of $G, f$ into Eq. (3.48) with $\xi=0$, and working as in the proof of Proposition 3.1, we readily obtain that $P_{m \mid p q}$ has the expression (3.47).

\section{Proof of Lemma 2.1. Connections with Propositions 3.1 and 3.2}

We begin with the

Proof of Lemma 2.1. Let us first prove the uniqueness of the polynomial fulfilling (2.17) for all functions $F$. To this purpose we consider any polynomial $P$ fulfilling (2.17), and show that $P\left(\left(\nu_{h \ell}\right), \rho\right)$ is uniquely determined for all $\nu_{h \ell} \in \mathbf{R}$ and $\rho \in(0,+\infty)$, a fact yielding the thesis. To prove this fact, given $\left(\nu_{h \ell}\right), \rho$ as above we define a function

$$
\begin{aligned}
F & =F_{m,\left(\nu_{h \ell}\right), \rho}: \mathbf{R}^{2} \rightarrow \mathbf{R} \\
F_{m,\left(\nu_{h \ell}\right), \rho}(u, \xi) & :=\sum_{\substack{1 \leq h \leq m \\
0 \leq \ell \leq m-h}} \nu_{h \ell} \frac{(u-\rho)^{h}(\xi-\ln \rho)^{\ell}}{h ! \ell !} .
\end{aligned}
$$


Then, it is found that

$$
\frac{\partial^{h+\ell} F_{m,\left(\nu_{h \ell}\right), \rho}}{\partial u^{h} \partial \xi^{\ell}}(\rho, \ln \rho)=\nu_{h \ell}, \quad \frac{\partial^{m} F_{m,\left(\nu_{h \ell}\right), \rho}}{\partial \xi^{m}}(\rho, \ln \rho)=0
$$

these results, with Eq. (2.17) for $P$, imply

$$
P\left(\left(\nu_{h \ell}\right), \rho\right)=\left.\frac{d^{m}}{d \xi^{m}}\right|_{\xi=\ln \rho} F_{m,\left(\nu_{h \ell}\right), \rho}\left(e^{\xi}, \xi\right),
$$

and so $P$ is uniquely determined.

The existence of the polynomial $P=P_{m}$ fulfilling Eq. (2.17), its form (2.18) and Eq. (2.21) can be proved in a single step. In fact, it is easy to check that the polynomial

$$
P_{m}\left(\left(\nu_{j \ell}\right), \rho\right):=\sum_{\substack{1 \leq j \leq m \\ 0 \leq \ell \leq m-j}} P_{m j \ell} \nu_{j \ell} \rho^{j}
$$

fulfills (2.17) for each $m \in \mathbf{N}_{0}$, if the coefficients $\left(P_{m j \ell}\right)$ satisfy the recursion relations (2.21). Let us consider these coefficients more closely. From the recursion relations, it is clear that they are integers. By construction, we have

$$
P_{m j \ell}=P_{m}\left(\nu_{j^{\prime} \ell^{\prime}}=\delta_{j^{\prime} j} \delta_{\ell^{\prime} \ell}, \rho=1\right) ;
$$

the r.h.s. is given by Eq. (4.3) for $P=P_{m}$, considering the derivative at $\xi=0$ and the function $F_{m,\left(\nu_{j^{\prime} \ell^{\prime}}=\delta_{j^{\prime} j} \delta_{\ell^{\prime} \ell}\right), \rho=1}(u, \xi)=(u-1)^{j} \xi^{\ell} /(j ! \ell !)$. In conclusion

$$
P_{m j \ell}=\left.\frac{d^{m}}{d \xi^{m}}\right|_{\xi=0} \frac{\left(e^{\xi}-1\right)^{j} \xi^{\ell}}{j ! \ell !},
$$

and this proves Eq. (2.19). Finally, let us write $\left(e^{\xi}-1\right)^{j}=$ $\sum_{r=1}^{j}\left(\begin{array}{l}j \\ r\end{array}\right) e^{r \xi}(-1)^{j-r}$ and expand $e^{r \xi}$ about $\xi=0$; inserting the expansion in the previous equation, and isolating the coefficient of $\xi^{m}$ we obtain the expression (2.20) for $P_{m j \ell}$.

Now, let us point out a relation between the coefficients in the general formulae for the derivation of a composite function and the coefficients $P_{m j \ell}$ of the polynomials $P_{m}$ (an equivalent formulation of the forthcoming Eq. (4.5) for $\ell=0$ can be found in [5]).

Lemma 4.1. Consider the coefficients $P_{m \mid p}$ and $P_{m \mid p q}$ in Eqs. (3.30) (3.46) for the derivation of a composite function, in the real and complex 
cases respectively. These are related to the coefficients $P_{m j \ell}$ of the polynomials $P_{m}$ by

$$
\begin{gathered}
\sum_{p \in D_{j, m-\ell}} P_{m \mid p}=P_{m j \ell} \\
\sum_{(p, q) \in \mathrm{D}_{h k, m-\ell}} P_{m \mid p q}=\left(\begin{array}{c}
h+k \\
h
\end{array}\right) P_{m, h+k, \ell} .
\end{gathered}
$$

Proof. We will derive in more detail Eq. (4.6); the argument yielding to (4.5) is even simpler. Consider any $C^{m}$ function

$$
G: \mathbf{C} \times \mathbf{R} \rightarrow \mathbf{R}, \quad(z, \xi) \rightarrow G(z, \xi),
$$

and apply the "1-dimensional" formula (3.48) with $f: \mathbf{R} \rightarrow \mathbf{R}, f(\xi):=e^{\xi}$; this gives

$$
\begin{aligned}
& \frac{d^{m}}{d \xi^{m}}\left(G\left(e^{\xi}, \xi\right)\right)=\sum_{\substack{h^{\prime}, k^{\prime} \geq 0,1 \leq h^{\prime}+k^{\prime} \leq m \\
0 \leq \ell \leq m-h^{\prime}-k^{\prime}}} \frac{\partial^{h^{\prime}+k^{\prime}+\ell^{\prime}} G}{\partial z^{h^{\prime}} \partial \bar{z}^{k^{\prime}} \partial \xi^{\prime}}\left(e^{\xi}, \xi\right) \\
& \times\left(\sum_{(p, q) \in \mathrm{D}_{h^{\prime} k^{\prime}, m-\ell^{\prime}}} P_{m \mid p q}\right) e^{\left(h^{\prime}+k^{\prime}\right) \xi}+\frac{\partial^{m} G}{\partial \xi^{m}}\left(e^{\xi}, \xi\right) .
\end{aligned}
$$

Let us choose, in particular,

$$
G(z, \xi):=\frac{(z-1)^{h}(\bar{z}-1)^{k} \xi^{\ell}}{h ! k ! \ell !}
$$

for fixed $h, k, \ell \geq 0$ with $1 \leq h+k \leq m$ and $0 \leq \ell \leq m-h-k$; we apply Eq. (4.8) with this $G$ at $\xi=0$. The l.h.s. of (4.8) gives the derivative at $\xi=0$ of $G\left(e^{\xi}, \xi\right)=\left(e^{\xi}-1\right)^{h+k} \xi^{\ell} /(h ! k ! \ell !) ;$ in the r.h.s. the only nonzero derivative is $\left(\partial^{h+k+\ell} G / \partial z^{h} \partial \bar{z}^{k} \partial \xi^{\ell}\right)(1,0)=1$, and thus

$$
\left.\frac{d^{m}}{d \xi^{m}}\right|_{\xi=0} \frac{\left(e^{\xi}-1\right)^{h+k} \xi^{\ell}}{h ! k ! \ell !}=\sum_{(p, q) \in \mathrm{D}_{h k, m-\ell}} P_{m \mid p q} .
$$

By comparison with Eq. (2.19), we see that the 1.h.s. in this equation equals $\left(\begin{array}{c}h+k \\ h\end{array}\right) P_{m, h+k, \ell} ;$ so, Eq. (4.6) is proved.

Eq. (4.5) is derived similarly, applying formula (3.46) to $f(\xi):=e^{\xi}$ and $G: \mathbf{R}^{2} \rightarrow \mathbf{R}, G(u, \xi):=(u-1)^{j} \xi^{\ell} /(j ! \ell !)$. 


\section{More on tensor functional spaces. Some inequalities}

We always consider spaces of complex tensor-valued functions; the real analogues can be regarded as subspaces of the complex ones.

Hölder type inequalities. $L^{p}$ spaces of functions $T: \mathbf{R}^{d} \mapsto \otimes \otimes^{\ell} \mathbf{C}^{d}$ have been already introduced, with the norms $\|T\|_{L^{p}}:=\||T|\|_{L^{p}}$ (see Eqs. (2.2-2.5)).

Let $h \in \mathbf{N}_{0}, \ell_{1}, \ldots, \ell_{h} \in \mathbf{N}$ and $p_{1}, \ldots, p_{h}, r \in[1,+\infty]$ be such that $1 / p_{1}+\ldots+1 / p_{h}=1 / r$; further, consider the tensor-valued functions $T_{1} \in L^{p_{1}}\left(\mathbf{R}^{d}, \otimes^{\ell_{1}} \mathbf{C}\right), \ldots, T_{h} \in L^{p_{h}}\left(\mathbf{R}^{d}, \otimes^{\ell_{h}} \mathbf{C}\right)$. Then $T_{1} \otimes \ldots \otimes T_{h}$, $T_{1} \vee \ldots \vee T_{h} \in L^{r}\left(\mathbf{R}^{d}, \otimes^{\ell_{1}+\ldots+\ell_{h}} \mathbf{C}^{d}\right)$, and

$$
\left\|T_{1} \otimes \ldots \otimes T_{h}\right\|_{L^{r}},\left\|T_{1} \vee \ldots \vee T_{h}\right\|_{L^{r}} \leq\left\|T_{1}\right\|_{L^{p_{1}}} \ldots\left\|T_{h}\right\|_{L^{p_{h}}}
$$

these Hölder type inequalities follow readily from the classical Hölder inequality and from Eq. (3.9) for the tensor norms $\mid$.

Fourier transform and Sobolev spaces. We denote with $\mathcal{F}$ : $\mathcal{S}^{\prime}\left(\mathbf{R}^{d}, \mathbf{C}\right) \rightarrow \mathcal{S}^{\prime}\left(\mathbf{R}^{d}, \mathbf{C}\right)$ the Fourier transform for tempered distributions; when using this, we write $k=\left(k_{1}, \ldots, k_{d}\right)$ for the running variable on $\mathbf{R}^{d}$ and $\mathrm{k}: \mathbf{R}^{d} \rightarrow \mathbf{R}^{d}$ for the identity mapping of this space into itself. We normalise $\mathcal{F}$ so that $(\mathcal{F} f)(k)=(2 \pi)^{-d / 2} \int_{\mathbf{R}^{d}} d x e^{-i k \bullet x} f(x)$ for $f$ in $L^{1}$.

For $m \in \mathbf{N}$, we denote with $\mathcal{S}^{\prime}\left(\mathbf{R}^{d}, \otimes^{m} \mathbf{C}^{d}\right)$ the space of families $T=\left(T_{\lambda}\right)_{\lambda \in\{1, \ldots, d\}^{m}}$ where each component is in $\mathcal{S}^{\prime}\left(\mathbf{R}^{d}, \mathbf{C}\right)$. We extend componentwisely the Fourier transform to tensor valued tempered distributions, setting

$$
\mathcal{F} T:=\left(\mathcal{F} T_{\lambda}\right)_{\lambda \in\{1, \ldots, d\}^{m}}
$$

for each $T$ as before. For $f \in \mathcal{S}^{\prime}\left(\mathbf{R}^{d}, \mathbf{C}\right)$ and $m \in \mathbf{N}$, the Fourier representation of distributional derivatives can be written as

$$
\nabla^{m} f=\mathcal{F}^{-1}\left(\otimes^{m}(i \mathrm{k}) \mathcal{F} f\right)
$$

where $\otimes^{m}(i \mathrm{k}):=(i \mathrm{k}) \otimes \ldots \otimes(i \mathrm{k})(m$ times $)$. This fact, with the preservation of the $L^{2}$ norm under $\mathcal{F}^{-1}$, implies

$$
\left\|\nabla^{m} f\right\|_{L^{2}}=\left\|\otimes^{m}(i \mathrm{k}) \mathcal{F} f\right\|_{L^{2}}=\left\||\mathrm{k}|^{m} \mathcal{F} f\right\|_{L^{2}},
$$

whenever one of the above three functions is in $L^{2}$; the second equality follows from the fact that the tensor norm $\left|\otimes^{m}(i k)\right|$ equals $|k|^{m}=$ ${\sqrt{k_{1}^{2}+\ldots+k_{d}^{2}}}^{m}$ for each $k \in \mathbf{R}^{d}$ (recall Eq. (3.9)). Due to these facts 
and to the equality

$$
\left(1+|\mathrm{k}|^{2}\right)^{n}=\sum_{m=0}^{n}\left(\begin{array}{c}
n \\
m
\end{array}\right)|\mathrm{k}|^{2 m},
$$

we see from Eqs. (2.7) (2.8) that

$$
\begin{gathered}
H^{n}\left(\mathbf{R}^{d}, \mathbf{C}\right)=\left\{f \in \mathcal{S}^{\prime}\left(\mathbf{R}^{d}, \mathbf{C}\right) \mid{\sqrt{1+|\mathrm{k}|^{2}}}^{n} \mathcal{F} f \in L^{2}\left(\mathbf{R}^{d}, \mathbf{C}\right)\right\}, \\
\|f\|_{n}=\left\|{\sqrt{1+|\mathrm{k}|^{2}}}^{n} \mathcal{F} f\right\|_{L^{2}}
\end{gathered}
$$

thus, the conventional definition of Bessel potential spaces is recovered. We note that Eqs. (5.6)-(5.7) also make sense for non integer $n$, and could be written in terms of the operator $\sqrt{1-\Delta}^{n}:=\mathcal{F}^{-1}{\sqrt{1+|\mathrm{k}|^{2}}}^{n} \mathcal{F}$.

Hausdorff-Young inequality. We recall the definition (2.13) for the function $E$. Let $p \in[1,2], r \in[2,+\infty]$ be such that $1 / p+1 / r=1$. Further, let $m \in \mathbf{N}$; for all $T \in L^{p}\left(\mathbf{R}^{d}, \otimes^{m} \mathbf{C}^{d}\right)$, it is $\mathcal{F}^{-1} T \in L^{r}\left(\mathbf{R}^{d}, \otimes^{m} \mathbf{C}\right)$ and

$$
\left\|\mathcal{F}^{-1} T\right\|_{L^{r}} \leq C_{r d}\|T\|_{L^{p}}, \quad C_{r d}:=\frac{1}{(2 \pi)^{d / 2-d / r}}\left(\frac{E(1 / r)}{E(1-1 / r)}\right)^{d / 2}
$$

furthermore, the above constant is the sharp one for the written inequality. In the case $m=0$, where $T$ is $\mathbf{C}$-valued, this result is classic (see Beckner's paper [3], or [12]; these give a slightly different expression for $C_{r d}$ due to a different normalisation for the Fourier transform). For $m=1,2,3, \ldots$, the above statement follows from the Marcinkiewicz-Zygmund theorem on the componentwise vector extensions of operators between $L^{p}$ spaces (see, e.g., [7], Ch.5, Theor.2.7; it must be noted that the norm (2.2) we use on $\otimes^{m} \mathbf{C}^{d}$ is a Hilbertian, $\ell^{2}$-type norm, as required by the theorem. We acknowledge L. Colzani for pointing out to us the cited result, and W. Beckner for general indications on the vectorial extensions of the Hausdorff-Young inequality).

An interpolation inequality. We rephrase in tensor notations a well known inequality; the aim is to point out the absence of $d$-dependent constants.

Proposition 5.1. Let $m \in \boldsymbol{N}, f \in L^{2}\left(\boldsymbol{R}^{d}, \boldsymbol{C}\right)$ such that $\nabla^{m} f \in$ $L^{2}\left(\boldsymbol{R}^{d}, \otimes^{m} \boldsymbol{C}\right)$. Then, for $\ell \in\{0, \ldots, m\}$, it is $\nabla^{\ell} f \in L^{2}\left(\boldsymbol{R}^{d}, \otimes^{\ell} \boldsymbol{C}\right)$ and

$$
\left\|\nabla^{\ell} f\right\|_{L^{2}} \leq\|f\|_{L^{2}}^{1-\ell / m}\left\|\nabla^{m} f\right\|_{L^{2}}^{\ell / m} \leq\left(1-\frac{\ell}{m}\right)\|f\|_{L^{2}}+\frac{\ell}{m}\left\|\nabla^{m} f\right\|_{L^{2}}
$$

(intending $\ell / m:=1$ for $\ell=m=0$ and $0^{t}:=0$ for each $t$ ). 
Proof. Let us prove that $\nabla^{\ell} f$ is $L^{2}$ and satisfies the first inequality (5.9). Due to (5.4), it suffices to show that $|\mathrm{k}|^{\ell} \mathcal{F} f \in L^{2}\left(\mathbf{R}^{d}, \mathbf{C}\right)$, and

$$
\left\||\mathrm{k}|^{\ell} \mathcal{F} f\right\|_{L^{2}} \leq\|\mathcal{F} f\|_{L^{2}}^{1-\ell / m}\left\||\mathrm{k}|^{m} \mathcal{F} f\right\|_{L^{2}}^{\ell / m}
$$

the desired result follows writing $|\mathrm{k}|^{\ell}|\mathcal{F} f|=\left(|\mathcal{F} f|^{1-\ell / m}\right)\left(|\mathrm{k}|^{\ell}|\mathcal{F} f|^{\ell / m}\right)$ and using the Hölder inequality \|\|$_{L^{2}} \leq\|\|_{L^{p}}\|\|_{L^{q}}$, with $1 / p=1 / 2-\ell /(2 m)$, $1 / q=\ell /(2 m)$.

The second inequality (5.9) follows trivially from the first one, due to the Young inequality $v^{1-\theta} w^{\theta} \leq(1-\theta) v+\theta w(v, w \in[0,+\infty), \theta \in[0,1])$.

A Gagliardo type inequality. The structure of the inequality is familiar, but little seems available about the constant appearing therein. Here we propose an explicit (probably non sharp) estimate for the constant, based on the use of Fourier methods and the Hausdorff-Young inequality (5.8). All derivatives in the forthcoming statement are intended in the distributional sense.

Proposition 5.2. Let $\ell, m, a \in N, \ell \leq m, a>d / 2$; intend $m / \ell:=1$ if $\ell=m=0$. If $f \in H^{a}\left(\boldsymbol{R}^{d}, \boldsymbol{C}\right)$ and $\nabla^{m} f \in L^{2}\left(\boldsymbol{R}^{d}, \otimes^{m} C^{d}\right)$, then $\nabla^{\ell} f \in L^{2 m / \ell}\left(\boldsymbol{R}^{d}, \otimes^{\ell} \boldsymbol{C}\right)$ and

$$
\left\|\nabla^{\ell} f\right\|_{L^{2 m / \ell}} \leq\left(\frac{E(\ell /(2 m))}{E(1-\ell /(2 m))}\right)^{d / 2}\left(S_{a d}\|f\|_{a}\right)^{1-\ell / m}\left\|\nabla^{m} f\right\|_{L^{2}}^{\ell / m}
$$

where $E$ is defined as in (2.13) and $S_{a d}$ is the constant (2.9).

Proof. We put

$$
r:=\frac{2 m}{\ell}, \quad p:=\frac{2 m}{2 m-\ell}, \quad s:=\frac{2 m}{m-\ell}
$$

(intending $s:=\infty$ if $m=\ell \neq 0 ; r:=s:=2$ and $p:=1$ if $m=\ell=0$ ). Then $r, s \in[2,+\infty], p \in[1,2]$ and

$$
\frac{1}{p}+\frac{1}{r}=1, \quad \frac{1}{r}+\frac{1}{s}=\frac{1}{2}, \quad \frac{2}{s}+\frac{1}{r}=\frac{1}{p} .
$$

By Eq. (5.3) and the Hausdorff-Young inequality (5.8), we have

$$
\begin{gathered}
\left\|\nabla^{\ell} f\right\|_{L^{r}}=\left\|\mathcal{F}^{-1}\left(\otimes^{\ell}(i \mathrm{k}) \mathcal{F} f\right)\right\|_{L^{r}} \leq C_{r d}\left\|\otimes^{\ell}(i \mathrm{k}) \mathcal{F} f\right\|_{L^{p}}= \\
=\frac{1}{(2 \pi)^{d / s}}\left(\frac{E(1 / r)}{E(1-1 / r)}\right)^{d / 2}\left\||\mathrm{k}|^{\ell} \mathcal{F} f\right\|_{L^{p}},
\end{gathered}
$$


provided that the last function be $L^{p}$. To prove this and the rest, we write

$$
|\mathrm{k}|^{\ell}|\mathcal{F} f|=\frac{1}{\left(1+|\mathrm{k}|^{2}\right)^{a / s}}\left(\left(1+|\mathrm{k}|^{2}\right)^{a / s}|\mathcal{F} f|^{2 / s}\right)\left(|\mathrm{k}|^{\ell}|\mathcal{F} f|^{2 / r}\right)
$$

and apply the Hölder inequality to the above three factors, recalling that $1 / s+1 / s+1 / r=1 / p ;$ this gives

$\left\||\mathrm{k}|^{\ell}|\mathcal{F} f|\right\|_{L^{p}} \leq\left\|\frac{1}{\left(1+|\mathrm{k}|^{2}\right)^{a / s}}\right\|_{L^{s}}\left\|\left(1+|\mathrm{k}|^{2}\right)^{a / s}|\mathcal{F} f|^{2 / s}\right\|_{L^{s}}\left\||\mathrm{k}|^{\ell}|\mathcal{F} f|^{2 / r}\right\|_{L^{r}}$

Let us show that the norms of the three factors are finite, and compute them. We have

$$
\left\|\frac{1}{\left(1+|\mathrm{k}|^{2}\right)^{a / s}}\right\|_{L^{s}}=\left(\int_{\mathbf{R}^{d}} \frac{d k}{\left(1+|k|^{2}\right)^{a}}\right)^{1 / s}=(2 \pi)^{d / s} S_{a d}^{2 / s} ;
$$

$$
\begin{aligned}
\left\|\left(1+|\mathrm{k}|^{2}\right)^{a / s}|\mathcal{F} f|^{2 / s}\right\|_{L^{s}} & =\left(\int_{\mathbf{R}^{d}} d k\left(1+|k|^{2}\right)^{a}|\mathcal{F} f|^{2}\right)^{1 / s}=\|f\|_{a}^{2 / s} \\
\left\||\mathrm{k}|^{\ell}|\mathcal{F} f|^{2 / r}\right\|_{L^{r}} & =\left(\int_{\mathbf{R}^{d}} d k|k|^{\ell r}|\mathcal{F} f|^{2}\right)^{1 / r} \\
& =\left(\int_{\mathbf{R}^{d}} d k|k|^{2 m}|\mathcal{F} f|^{2}\right)^{1 / r} \\
& =\left\|\otimes^{m}(i \mathrm{k}) \mathcal{F} f\right\|_{L^{2}}^{2 / r} \\
& =\left\|\nabla^{m} f\right\|_{L^{2}}^{2 / r}
\end{aligned}
$$

(some intermediate steps where integrals appear do not make sense for $s=\infty$ or $r=\infty$, but the final results also cover these cases).

Now, we insert Eqs. (5.17-5.19) into (5.16), and the result into Eq. (5.14); after explicitating $r$ and $s$, we readily get the thesis (5.11).

The Adams-Frazier inequality. Again, we discuss an inequality whose structure is known [1] but for which the constants were not previously estimated, to the best of our knowledge. As in the previous subsection, we intend derivatives in the distributional sense.

Proposition 5.3. Let $h, k \in \boldsymbol{N}, h+k \geq 1$ and $i_{1}, \ldots, i_{h}, g_{1}, \ldots, g_{k} \in \boldsymbol{N}_{0}$; put $m:=i_{1}+\ldots+i_{h}+g_{1}+\ldots+g_{k}$. Furthermore, let $a>d / 2$ and consider a function $f \in H^{a}\left(\boldsymbol{R}^{d}, \boldsymbol{C}\right)$ with $\nabla^{m} f \in L^{2}\left(\boldsymbol{R}^{d}, \boldsymbol{C}\right)$. Then $\nabla^{i_{1}} f, \ldots, \nabla^{g_{k}} f$ are ordinary functions, the product $\vee$ written below is $L^{2}$, and

$$
\left\|\nabla^{i_{1}} f \vee \ldots \vee \nabla^{i_{h}} f \vee \overline{\nabla^{g_{1}} f} \ldots \vee \overline{\nabla^{g_{k}} f}\right\|_{L^{2}}
$$




$$
\leq U_{m, h+k, d}\left(S_{a d}\|f\|_{a}\right)^{h+k-1}\left\|\nabla^{m} f\right\|_{L^{2}},
$$

where $U_{m, h+k, d}(\leq 1)$ is defined as in $(2.14)$.

Proof. The statement is trivial if $h+k=1$; in the sequel $h+k \geq 2$. We put

$$
p_{u}:=\frac{2 m}{i_{u}}, \quad q_{v}:=\frac{2 m}{g_{v}}
$$

for $u \in\{1, \ldots, h\}, v \in\{1, \ldots, k\}$; then $p_{u}, q_{v} \in[2,+\infty)$ and

$$
\sum_{u=1}^{h} \frac{1}{p_{u}}+\sum_{v=1}^{k} \frac{1}{q_{v}}=\frac{1}{2} .
$$

Thus, from the Hölder inequality we get

$$
\left\|\nabla^{i_{1}} f \vee \ldots \vee \overline{\nabla^{g_{k}} f}\right\|_{L^{2}} \leq\left\|\nabla^{i_{1}} f\right\|_{L^{p_{1}}} \ldots\left\|\overline{\bar{\nabla}^{g_{k}} f}\right\|_{L^{q_{k}}}
$$

provided that all norms in the r.h.s. be finite. In fact it is so, and the Gagliardo inequality with $\ell=i_{u}$ or $\ell=g_{v}$ and $m$ as above gives

$$
\left\|\nabla^{i_{u}} f\right\|_{L^{p_{u}}} \leq\left(\frac{E\left(i_{u} /(2 m)\right)}{E\left(1-i_{u} /(2 m)\right)}\right)^{d / 2}\left(S_{a d}\|f\|\right)_{a}^{1-i_{u} / m}\left\|\nabla^{m} f\right\|_{L^{2}}^{i_{u} / m}
$$

$\left\|\overline{\nabla^{g_{v}} f}\right\|_{L^{q_{v}}}=\left\|\nabla^{g_{v}} f\right\|_{L^{q_{v}}} \leq\left(\frac{E\left(g_{v} /(2 m)\right)}{E\left(1-g_{v} /(2 m)\right)}\right)^{d / 2}\left(S_{a d}\|f\|\right)_{a}^{1-g_{v} / m}\left\|\nabla^{m} f\right\|_{L^{2}}^{g_{v} / m}$.

Inserting these estimates into (5.23) and recalling that $i_{1}+\ldots+g_{k}=m$, we readily get

$$
\begin{gathered}
\left\|\nabla^{i_{1}} f \vee \ldots \vee \overline{\nabla^{g_{k}} f}\right\|_{L^{2}} \leq \mathcal{U}_{i_{1} \ldots g_{k}, d}\left(S_{a d}\|f\|_{a}\right)^{h+k-1}\left\|\nabla^{m} f\right\|_{L^{2}}, \\
\mathcal{U}_{i_{1} \ldots g_{k}, d}:=\left(\Pi_{u=1}^{h} \frac{E\left(i_{u} /(2 m)\right)}{E\left(1-i_{u} /(2 m)\right)} \Pi_{v=1}^{k} \frac{E\left(g_{v} /(2 m)\right)}{E\left(1-g_{v} /(2 m)\right)}\right)^{d / 2}
\end{gathered}
$$

now, the proof is concluded if we show that

$$
\mathcal{U}_{i_{1} \ldots g_{k}, d} \leq U_{m, h+k, d} .
$$

To prove this, we put

$$
\mathcal{H}:(0,1)^{h+k} \rightarrow \mathbf{R},
$$




$$
\vartheta=\left(\vartheta_{1}, \ldots, \vartheta_{h+k}\right) \mapsto \mathcal{H}(\vartheta):=\sum_{w=1}^{h+k} \frac{\vartheta_{w}}{2} \ln \frac{\vartheta_{w}}{2}-\left(1-\frac{\vartheta_{w}}{2}\right) \ln \left(1-\frac{\vartheta_{w}}{2}\right)
$$

and denote with $\eta, \zeta_{(1)}, \ldots, \zeta_{(h+k)}$ the following points of the cube $(0,1)^{h+k}$ :

$$
\begin{aligned}
\eta:= & \left(\frac{i_{1}}{m}, \ldots, \frac{i_{h}}{m}, \frac{g_{1}}{m}, \ldots, \frac{g_{k}}{m}\right), \\
\zeta_{(1)}:= & \left(1-\frac{h+k-1}{m}, \frac{1}{m}, \ldots, \frac{1}{m}\right), \\
\zeta_{(2)}:= & \left(\frac{1}{m}, 1-\frac{h+k-1}{m}, \frac{1}{m}, \ldots, \frac{1}{m}\right), \ldots, \\
& \zeta_{(h+k)}:=\left(\frac{1}{m}, \ldots, \frac{1}{m}, 1-\frac{h+k-1}{m}\right) ;
\end{aligned}
$$

then

$$
\begin{gathered}
\frac{d}{2} \mathcal{H}(\eta)=\ln \mathcal{U}_{i_{1} \ldots g_{k}, d}, \\
\frac{d}{2} \mathcal{H}\left(\zeta_{(1)}\right)=\ldots=\frac{d}{2} \mathcal{H}\left(\zeta_{(h+k)}\right)=\ln U_{m, h+k, d} .
\end{gathered}
$$

It is readily cheched that $\mathcal{H}$ is a convex function (its Hessian is a diagonal, positive defined matrix); on the other hand

$$
\begin{gathered}
\eta=\sum_{w=1}^{h+k} t_{w} \zeta_{(w)}, \\
t_{1}:=\frac{i_{1}-1}{m-h-k}, \ldots, t_{h}:=\frac{i_{h}-1}{m-h-k}, \\
t_{h+1}:=\frac{g_{1}-1}{m-h-k}, \ldots, t_{h+k}:=\frac{g_{k}-1}{m-h-k}
\end{gathered}
$$

(intending $t_{1}:=\ldots=t_{h+k}:=1 /(h+k)$ if $m=h+k$; this case occurs if and only if $i_{1}=\ldots=g_{k}=1$ ). By construction $t_{w} \geq 0$ for all $w$, and $\sum_{w=1}^{h+k} t_{w}=1$. Thus, by the convexity of $\mathcal{H}$ and (5.30),

$$
\ln \mathcal{U}_{i_{1} \ldots g_{k}, d}=\frac{d}{2} \mathcal{H}(\eta) \leq \frac{d}{2} \sum_{w=1}^{h+k} t_{w} \mathcal{H}\left(\zeta_{(w)}\right)=\ln U_{m, h+k, d} .
$$

This gives Eq. (5.27), and concludes the proof. 


\section{Proofs of Propositions 2.4 and 2.7}

We work out in detail the complex case only, corresponding to Proposition 2.7; the real case can be treated similarly (and is in fact simpler).

The main step towards the proof of Proposition 2.7 is to estimate the norms $\left\|\nabla^{m}(G(f, \mathrm{x}))-\nabla^{m}(G(0, \mathrm{x}))\right\|_{L^{2}}(0 \leq m \leq n)$ under the given assumptions on $G$ and $f$. Formula (3.46) for the derivatives of a composite function is essential for this purpose; the result is the following.

Proposition 6.1. Let $n, a \in \boldsymbol{N}$ and $a>d / 2$. Consider a function $G$ : $B_{\boldsymbol{C}}(0, r) \times \boldsymbol{R}^{d} \rightarrow \boldsymbol{C}$ with the $\Pi^{n}$ property, such that $G(0, \mathrm{x}) \in H^{n}\left(\boldsymbol{R}^{d}, \boldsymbol{C}\right)$, and a function $f \in H^{n}\left(\boldsymbol{R}^{d}, \boldsymbol{C}\right) \cap H_{r}^{a}\left(\boldsymbol{R}^{d}, \boldsymbol{R}\right)$.

Then $G(f, \mathrm{x}) \in H^{n}\left(\boldsymbol{R}^{d}, \boldsymbol{C}\right)$; furthermore, it is

$$
\begin{gathered}
\|G(f, \mathrm{x})-G(0, \mathrm{x})\|_{L^{2}} \leq b_{0}\left(G, S_{a d}\|f\|_{a}\right)\|f\|_{L^{2}}, \\
\left\|\nabla^{m}(G(f, \mathrm{x}))-\nabla^{m}(G(0, \mathrm{x}))\right\|_{L^{2}} \\
\leq \beta_{m d}\left(G, S_{a d}\|f\|_{a}\right)\left\|\nabla^{m} f\right\|_{L^{2}}+\left(b_{m d}\left(G, S_{a d}\|f\|_{a}\right)\right. \\
\left.\quad+b_{m}\left(G, S_{a d}\|f\|_{a}\right)\right)\|f\|_{L^{2}} \quad(1 \leq m \leq n),
\end{gathered}
$$

where $b_{0}, b_{m}, \beta_{m d}, b_{m d}$ are defined as in Eqs. (2.27), (2.29) and (2.30).

Proof. Step 1: $G(f, \mathrm{x}) \in L^{2}\left(\boldsymbol{R}^{d}, C\right)$, and Eq. (6.1) holds. By our assumptions $G(0, \mathrm{x})$ is $L^{2}$; thus, we must show that $G(f, \mathrm{x})-G(0, \mathrm{x})$ is $L^{2}$, and that its norm has the bound (6.1). Recalling the definition (2.38) we see that, for $\rho \in[0, r), z \in \bar{B}_{\mathbf{C}}(0, \rho)$ and $x \in \mathbf{R}^{d}$,

$$
|G(z, x)-G(0, x)| \leq b_{0}(G, \rho)|z| \text {. }
$$

Due to (2.10), we can apply this with $z=f(x), \rho=S_{a d}\|f\|_{a}$; thus, integrating over $x$ (the squares of) both sides, we obtain Eq. (6.1).

Step 2 (for $n \geq 1)$. It is $\nabla^{m}(G(f, \mathrm{x})) \in L^{2}\left(\boldsymbol{R}^{d}, \boldsymbol{C}\right)$ and (6.2) holds for $1 \leq m \leq n$, if $f \in H^{n}\left(\boldsymbol{R}^{d}, \boldsymbol{C}\right) \cap H_{r}^{a}\left(\boldsymbol{R}^{d}, \boldsymbol{R}\right) \cap C^{n}\left(\boldsymbol{R}^{d}, C\right)$. The supplementary assumption that $f$ be $C^{n}$ allows us to use Eq. (3.46) for the derivatives of a composite function, in the usual (i.e., not distributional) sense (next, the $C^{n}$ assumption on $f$ will be removed by appropriate density arguments, see Step 3). For the sake of brevity, let us put

$$
\mathcal{G}: B_{\mathbf{C}}(0, r) \times \mathbf{R}^{d} \rightarrow \mathbf{C}, \quad \mathcal{G}(z, x):=G(z, x)-G(0, x)
$$

then $\mathcal{G}$ also possesses the $\Pi^{n}$ property. By our assumptions, $\nabla^{m}(G(0, \mathrm{x}))$ is $L^{2}$; we will show that $\nabla^{m}(\mathcal{G}(f, \mathrm{x}))$ is also $L^{2}$, with its $L^{2}$ norm bounded by the r.h.s. of (6.2). Let us express $\nabla^{m}(\mathcal{G}(f, \mathrm{x}))$ according to Eq. (3.46) 
(with $G$ replaced by $\mathcal{G}$ ); this implies

$$
\begin{gathered}
\left\|\nabla^{m}(\mathcal{G}(f, \mathrm{x}))\right\|_{L^{2}} \leq \sum_{\substack{h, k \geq 0,1 \leq h+k \leq m \\
0 \leq \ell \leq m-h-k}}\left\|\partial^{h} \bar{\partial}^{k} \nabla^{\ell} \mathcal{G}(f, \mathrm{x})\right\|_{L^{\infty}} \\
\times \sum_{(p, q) \in \mathrm{D}_{h k, m-\ell}} P_{m \mid p q} \|\left(\vee^{p_{1}} \nabla^{1} f\right) \vee\left(\vee^{p_{2}} \nabla^{2} f\right) \ldots \\
\quad\left(\vee^{q_{1}} \overline{\nabla^{1} f}\right) \vee\left(\vee^{q_{2}} \overline{\nabla^{2} f}\right) \ldots\left\|_{L^{2}}+\right\| \nabla^{m} \mathcal{G}(f, \mathrm{x}) \|_{L^{2}},
\end{gathered}
$$

provided that all norms in the r.h.s. be finite (we have used the Hölder inequality for tensor fields, in the form $\|T \vee S\|_{L^{2}} \leq\|T\|_{L^{\infty}}\|S\|_{L^{2}}$ ).

Let $h, k, \ell$ be as in the above sum; then $\partial^{h} \bar{\partial}^{k} \nabla^{\ell} \mathcal{G}=\partial^{h} \bar{\partial}^{k} \nabla^{\ell} G$ (because the $z$-independent term $x \mapsto G(0, x)$ disappears on application of $\left.\partial^{h} \bar{\partial}^{k}\right)$. Recalling the definition (2.39) we see that, for $\rho \in[0, r), z \in \bar{B}_{\mathbf{C}}(0, \rho)$ and $x \in \mathbf{R}^{d}$,

$$
\left|\partial^{h} \bar{\partial}^{k} \nabla^{\ell} \mathcal{G}(z, x)\right|=\left|\partial^{h} \bar{\partial}^{k} \nabla^{\ell} G(z, x)\right| \leq \sigma_{h k \ell}(G, \rho) ;
$$

due to (2.10), we can apply this inequality with $z=f(x)$ and $\rho=S_{a d}\|f\|_{a}$, so

$$
\left\|\partial^{h} \bar{\partial}^{k} \nabla^{\ell} \mathcal{G}(f, \mathrm{x})\right\|_{L^{\infty}}=\sup _{x \in \mathbf{R}^{d}}\left|\partial^{h} \bar{\partial}^{k} \nabla^{\ell} G(f(x), x)\right| \leq \sigma_{h k \ell}\left(G, S_{a d}\|f\|_{a}\right) .
$$

Let us pass to the products

$$
\left(\vee^{p_{1}} \nabla^{1} f\right) \vee\left(\vee^{p_{2}} \nabla^{2} f\right) \ldots\left(\vee^{q_{1}} \overline{\nabla^{1} f}\right) \vee\left(\vee^{q_{2}} \overline{\nabla^{2} f}\right) \ldots
$$

with $(p, q) \in \mathrm{D}_{h k, m-\ell}$; any one of them can be reexpressed as

$$
\nabla^{i_{1}} f \vee \ldots \nabla^{i_{j}} f \vee \overline{\nabla^{g_{1}} f} \vee \ldots \vee \overline{\nabla^{g_{k}} f}
$$

where $i_{1} \leq i_{2} \leq \ldots \leq i_{j}, g_{1} \leq g_{2} \ldots \leq g_{k}$ are positive integers, determined in an obvious way by the sequences $(p, q)$. The above expression envolves $h+k$ factors, with total order $i_{1}+\ldots+g_{k}=m-\ell$, and its $L^{2}$ norm can be bounded by the Adams-Frazier inequality (5.20) in terms of $\left\|\nabla^{m-\ell} f\right\|_{L^{2}}$; the last norm can be interpolated with the second inequality (5.9). Summing 
up

$$
\begin{aligned}
\| & \left(\vee^{p_{1}} \nabla^{1} f\right) \vee\left(\vee^{p_{2}} \nabla^{2} f\right) \ldots\left(\vee^{p_{1}} \overline{\nabla^{1} f}\right) \vee\left(\vee^{p_{2}} \overline{\nabla^{2} f}\right) \ldots \|_{L^{2}} \\
& =\left\|\nabla^{i_{1}} f \vee \ldots \vee \overline{\nabla^{g_{k}} f}\right\|_{L^{2}} \\
& \leq U_{m-\ell, h+k, d}\left(S_{a d}\|f\|_{a}\right)^{h+k-1}\left\|\nabla^{m-\ell} f\right\|_{L^{2}} \\
& \leq U_{m-\ell, h+k, d}\left(S_{a d}\|f\|_{a}\right)^{h+k-1}\left(\frac{\ell}{m}\|f\|_{L^{2}}+\left(1-\frac{\ell}{m}\right)\left\|\nabla^{m} f\right\|_{L^{2}}\right)
\end{aligned}
$$

Finally, let us consider the term $\left\|\nabla^{m} \mathcal{G}(f, \mathrm{x})\right\|_{L^{2}}$; a straightforward generalization of Eq. (6.3) implies

$$
\left\|\nabla^{m} \mathcal{G}(f, \mathrm{x})\right\|_{L^{2}} \leq b_{m}\left(G, S_{a d}\|f\|_{a}\right)\|f\|_{L^{2}} .
$$

Let us insert the results (6.6) (6.7) (6.8) into Eq. (6.5); the sums over $(q, p) \in \mathrm{D}_{h k, m-\ell}$ of the coefficients $P_{m \mid q p}$ are related as in Eq. (4.6) to the coefficients $P_{m, h+k, \ell}$ of the polynomial $P_{m}$, and so

$$
\left\|\nabla^{m}(\mathcal{G}(f, \mathrm{x}))\right\|_{L^{2}} \leq\left(\sum_{\substack{h, k \geq 0,1 \leq h+k \leq m \\
0 \leq \ell \leq m-h-k}}\left(\begin{array}{c}
h+k \\
h
\end{array}\right) P_{m, h+k, \ell}\right.
$$

$$
\begin{aligned}
& \left.\times\left(1-\frac{\ell}{m}\right) U_{m-\ell, h+k, d} \sigma_{h k \ell}\left(G, S_{a d}\|f\|_{a}\right)\left(S_{a d}\|f\|_{a}\right)^{h+k-1}\right)\left\|\nabla^{m} f\right\|_{L^{2}} \\
& +\left(\sum_{\substack{h, k \geq 0,1 \leq h+k \leq m \\
0 \leq \ell \leq m-h-k}}\left(\begin{array}{c}
h+k \\
h
\end{array}\right) P_{m, h+k, \ell} \frac{\ell}{m} U_{m-\ell, h+k, d}\right. \\
& \left.\times \sigma_{h k \ell}\left(G, S_{a d}\|f\|_{a}\right)\left(S_{a d}\|f\|_{a}\right)^{h+k-1}\right)\|f\|_{L^{2}}+b_{m}\left(G, S_{a d}\|f\|_{a}\right)\|f\|_{L^{2}} .
\end{aligned}
$$

Let us compare this result with the definition $(2.40)$ of $\sigma_{j \ell}(G, \rho)$ in terms of the coefficients $\sigma_{h k \ell}(G, \rho)$, with $h+k=j$; this gives

$$
\begin{aligned}
\leq & \left(\sum_{\substack{1 \leq j \leq m \\
0 \leq \ell \leq m-j}} P_{m j \ell}\left(1-\frac{\ell}{m}\right) U_{m-\ell, j d} \sigma_{j \ell}\left(G, S_{a d}\|f\|_{a}\right)\left(S_{a d}\|f\|_{a}\right)^{j-1}\right)\left\|\nabla^{m} f\right\|_{L^{2}} \\
& +\left(\sum_{\substack{1 \leq j \leq m \\
0 \leq \ell \leq m-j}} P_{m j \ell} \frac{\ell}{m} U_{m-\ell, j d} \sigma_{j \ell}\left(G, S_{a d}\|f\|_{a}\right)\left(S_{a d}\|f\|_{a}\right)^{j-1}\right)\|f\|_{L^{2}} \\
& +b_{m}\left(G, S_{a d}\|f\|_{a}\right)\|f\|_{L^{2}} .
\end{aligned}
$$


From the expression (2.18) of the polynomial $P_{m}$ and the definitions (2.29) (2.30) of $\beta_{m d}, b_{m d}$ in terms of $P_{m}$, we see that the previous equation means

$$
\left\|\nabla^{m}(\mathcal{G}(f, \mathrm{x}))\right\|_{L^{2}} \leq \text { r.h.s. of Eq. (6.2) }
$$

as desired.

Step 3 (for $n \geq 1$ ). It is $\nabla^{m}(G(f, \mathrm{x})) \in L^{2}\left(\boldsymbol{R}^{d}, \boldsymbol{C}\right)$ and (6.2) holds for $1 \leq m \leq n$ and $f \in H^{n}\left(\boldsymbol{R}^{d}, \boldsymbol{C}\right) \cap H_{r}^{a}\left(\boldsymbol{R}^{d}, \boldsymbol{R}\right)$. Let $f \in H^{n} \cap H_{r}^{a}$, and put

$$
g:=G(f, \mathrm{x})-G(0, \mathrm{x}) .
$$

This is an $L^{2}$ function due to Step 1; the thesis follows if we show that $\nabla^{m} g$ is $L^{2}$ for $1 \leq m \leq n$, with the $L^{2}$ norm bounded by the r.h.s. of (6.2). As anticipated, a density argument will be employed to prove this.

In the sequel, $s$ is an index ranging over $\mathbf{N}$, and $\stackrel{s}{\rightarrow}$ indicates convergence for $s \rightarrow+\infty$. By standard density results, there is a sequence of functions $\left(f_{s}\right)$ such that

$$
f_{s} \in H^{n}\left(\mathbf{R}^{d}, \mathbf{C}\right) \cap H_{r}^{a}\left(\mathbf{R}^{d}, \mathbf{R}\right) \cap C^{n}\left(\mathbf{R}^{d}, \mathbf{C}\right), \quad\left\|f_{s}-f\right\|_{\max (n, a)} \stackrel{s}{\rightarrow} 0 .
$$

Let us put

$$
g_{s}:=G\left(f_{s}, \mathrm{x}\right)-G(0, \mathrm{x})
$$

then, due to Steps 1 and 2, we have

$$
\begin{gathered}
g_{s} \in L^{2}\left(\mathbf{R}^{d}, \mathbf{C}\right), \quad \nabla^{m} g_{s} \in L^{2}\left(\mathbf{R}^{d}, \otimes^{m} \mathbf{C}\right) \quad(1 \leq m \leq n) \\
\left\|g_{s}\right\|_{L^{2}} \leq b_{0}\left(G, S_{a d}\left\|f_{s}\right\|_{a}\right)\left\|f_{s}\right\|_{L^{2}} \stackrel{s}{\rightarrow} b_{0}\left(G, S_{a d}\|f\|_{a}\right)\|f\|_{L^{2}},
\end{gathered}
$$

$$
\begin{aligned}
\left\|\nabla^{m} g_{s}\right\|_{L^{2}} \leq & \beta_{m d}\left(G, S_{a d}\left\|f_{s}\right\|_{a}\right)\left\|\nabla^{m} f_{s}\right\|_{L^{2}} \\
& +b_{m d}\left(G, S_{a d}\left\|f_{s}\right\|_{a}\right)\left\|f_{s}\right\|_{L^{2}} \\
& +b_{m}\left(G, S_{a d}\left\|f_{s}\right\|_{a}\right)\|f\|_{L^{2}} \stackrel{s}{\rightarrow} \beta_{m d}\left(G, S_{a d}\|f\|_{a}\right)\left\|\nabla^{m} f\right\|_{L^{2}} \\
& +b_{m d}\left(G, S_{a d}\|f\|_{a}\right)\|f\|_{L^{2}} \\
& +b_{m}\left(G, S_{a d}\|f\|_{a}\right)\|f\|_{L^{2}} \quad(1 \leq m \leq n) ;
\end{aligned}
$$

the statements on the limits over $s$ follow from the continuity of the mappings $b_{0}, b_{m}, b_{m d}, \beta_{m d}$, and also imply

$$
\sup _{s}\left\|g_{s}\right\|_{L^{2}}<+\infty, \quad \sup _{s}\left\|\nabla^{m} g_{s}\right\|_{L^{2}}<+\infty \quad(1 \leq m \leq n) .
$$


Let us return to the function $g$ of Eq. (6.12), which is also $L^{2}$ due to Step 1 ; we claim that

$$
\left\|g_{s}-g\right\|_{L^{2}} \stackrel{s}{\rightarrow} 0 .
$$

In fact $g_{s}-g=G\left(f_{s}, \mathrm{x}\right)-G(f, \mathrm{x})$; to estimate this difference, we note that, by the Lagrange formula, for any radius $\rho \in[0, r)$ and all $z, z^{\prime} \in \bar{B}_{\mathbf{C}}(0, \rho)$, $x \in \mathbf{R}^{d}$, it is

$G\left(z^{\prime}, x\right)-G(z, x)=\int_{0}^{1} d t\left[\partial G\left((1-t) z+t z^{\prime}, x\right)\left(z^{\prime}-z\right)+\bar{\partial} G\left((1-t) z+t z^{\prime}, x\right)\left(\overline{z^{\prime}}-\bar{z}\right)\right]$,

whence

$$
\left|G\left(z^{\prime}, x\right)-G(z, x)\right| \leq\left(\sigma_{100}(G, \rho)+\sigma_{010}(G, \rho)\right)\left|z^{\prime}-z\right| .
$$

In particular, we choose $\rho$ such that $S_{a d}\|f\|_{a}<\rho$ and $S_{a d}\left\|f_{s}\right\|_{a}<\rho$ for $s$ large; applying Eq. (6.20) with $z=f(x)$ and $z^{\prime}=f_{s}(x)$, and integrating (the squares of) both sides over $x$, we get

$$
\left\|g_{s}-g\right\|_{L^{2}} \leq\left(\sigma_{100}(G, \rho)+\sigma_{010}(G, \rho)\right)\left\|f_{s}-f\right\|_{L^{2}} \stackrel{s}{\rightarrow} 0,
$$

thus proving (6.19). The rest of our argument depends on some known facts on weak convergence. Of course, Eq. (6.19) implies

$$
g_{s} \stackrel{s}{\rightarrow} g \quad \text { weakly in } L^{2}\left(\mathbf{R}^{d}, \mathbf{C}\right) .
$$

According to a general result on Sobolev spaces (see e.g. [6], Part 1, Sect.6, Lemma 6.2), statements (6.18) (6.21) are sufficient to infer that for $1 \leq m \leq n$

(6.22) $\nabla^{m} g \in L^{2}\left(\mathbf{R}^{d}, \otimes^{m} \mathbf{C}^{d}\right), \quad \nabla^{m} g_{s} \stackrel{s}{\rightarrow} \nabla^{m} g$ weakly in $L^{2}\left(\mathbf{R}^{d}, \otimes^{m} \mathbf{C}^{d}\right)$.

So, by the uniform boundedness theorem [21] we have

$$
\left\|\nabla^{m} g\right\|_{L^{2}} \leq \liminf _{s}\left\|\nabla^{m} g_{s}\right\|_{L^{2}}
$$

for $1 \leq m \leq n$; this fact, with Eq. (6.17), implies

$$
\left\|\nabla^{m} g\right\|_{L^{2}} \leq \text { r.h.s. of }(6.2)
$$

and the proof is concluded. (As a final remark, we would point out that the use of weak convergence in relation to Nemytskij operators was suggested in [19]). 
Finally, we are ready to give the

Proof of Proposition 2.7. From the previous Proposition, we already know that $G(f, \mathrm{x})$ is $H^{n}$; let us evaluate

$$
\|G(f, \mathrm{x})-G(0, \mathrm{x})\|_{n}=\sqrt{\sum_{m=0}^{n}\left(\begin{array}{c}
n \\
m
\end{array}\right)\left\|\nabla^{m}(G(f, \mathrm{x}))-\nabla^{m}(G(0, \mathrm{x}))\right\|_{L^{2}}^{2}} .
$$

Proposition 6.1 tells us that

$$
\left\|\nabla^{m}(G(f, \mathrm{x}))-\nabla^{m}(G(0, \mathrm{x}))\right\|_{L^{2}} \leq X_{m}+Y_{m} \quad(m=0, \ldots, n)
$$

$X_{0}:=b_{0}\left(G, S_{a d}\|f\|_{a}\right)\|f\|_{L^{2}}, X_{m}:=\beta_{m d}\left(G, S_{a d}\|f\|_{a}\right)\left\|\nabla^{m} f\right\|_{L^{2}}(1 \leq m \leq n)$,

$Y_{0}:=0, \quad Y_{m}:=\left(b_{m d}\left(G, S_{a d}\|f\|_{a}\right)+b_{m}\left(G, S_{a d}\|f\|_{a}\right)\right)\|f\|_{L^{2}}(1 \leq m \leq n)$.

Inserting this into Eq. (6.25) we get

$$
\begin{aligned}
\|G(f, \mathrm{x})-G(0, \mathrm{x})\|_{n} & \leq \sqrt{\sum_{m=0}^{n}\left(\begin{array}{c}
n \\
m
\end{array}\right)\left(X_{m}+Y_{m}\right)^{2}} \\
& \leq \sqrt{\sum_{m=0}^{n}\left(\begin{array}{c}
n \\
m
\end{array}\right) X_{m}^{2}}+\sqrt{\sum_{m=0}^{n}\left(\begin{array}{c}
n \\
m
\end{array}\right) Y_{m}^{2}} .
\end{aligned}
$$

On the other hand, the definitions of $X_{m}, Y_{m}$ imply

$$
\sqrt{\sum_{m=0}^{n}\left(\begin{array}{c}
n \\
m
\end{array}\right) X_{m}^{2}} \leq \max \left\{b_{0}\left(G, S_{a d}\|f\|_{a}\right), \beta_{m d}\left(G, S_{a d}\|f\|_{a}\right)_{m=1, \ldots, n}\right\}\|f\|_{n} ;
$$

$$
\sqrt{\sum_{m=0}^{n}\left(\begin{array}{c}
n \\
m
\end{array}\right) Y_{m}^{2}}=\sqrt{\sum_{m=1}^{n}\left(\begin{array}{c}
n \\
m
\end{array}\right)\left(b_{m d}\left(G, S_{a d}\|f\|_{a}\right)+b_{m}\left(G, S_{a d}\|f\|_{a}\right)\right)^{2}}\|f\|_{L^{2}}
$$

inserting (6.28) (6.29) into (6.27), we obtain the bound (2.31-2.33) on $\|G(f, \mathrm{x})-G(0, \mathrm{x})\|_{n}$; the weaker bound (2.34) follows trivially, since $\|f\|_{L^{2}} \leq$ $\|f\|_{n}$.

Comments and acknowledgments. The quantitative estimates in the present work are extended with little effort to Nemytskij operators on spaces $H^{n}\left(\mathbf{R}^{d}, \mathbf{R}^{\delta}\right.$ or $\left.\mathbf{C}^{\delta}\right)$, where $\delta$ is an arbitrary integer; here, this has not been done to keep notations as simple as possible. 
We again acknowledge W. Beckner, L. Colzani, G. Meloni and S. Paveri Fontana for useful indications. Also, we are grateful to an anonymous referee for some suggestions on the style of the paper.

This work was partly supported by the GNFM of Istituto Nazionale di Alta Matematica and by MIUR.

\section{Appendix A. Some facts about tensors}

Identities for permutation and symmetrization operators. For each $\ell$, we have defined the permutation operators $\mathcal{P}_{\sigma}$ and the symmetrization operator $\mathcal{S}$, sending $\otimes^{\ell} \mathbf{C}^{d}$ into itself (Eqs. (3.3)-(3.5)). For all $\ell$ and all permutations $\sigma, \sigma^{\prime} \in \ell$ !, it is readily checked that

$$
\begin{aligned}
& \mathcal{P}_{\sigma} \mathcal{P}_{\sigma^{\prime}}=\mathcal{P}_{\sigma \circ \sigma^{\prime}} \\
& \mathcal{S} \mathcal{P}_{\sigma}=\mathcal{P}_{\sigma} \mathcal{S}=\mathcal{S}
\end{aligned}
$$

From here, with elementary manipulations one infers the relations

$$
\mathcal{S}((\mathcal{S} T) \otimes U)=\mathcal{S}(T \otimes(\mathcal{S} U))=\mathcal{S}(T \otimes U)
$$

for all tensors $T \in \otimes^{\ell} \mathbf{C}^{d}, U \in \otimes^{m} \mathbf{C}^{d}$ (see e.g. [11], Chapter 4, in the proof of Proposition 5.7).

Proofs of the commutative and associative properties (3.7) of the symmetrized tensor product. The arguments are standard [11], and reported only for completeness. Let $T \in \otimes^{\ell} \mathbf{C}^{d}, U \in \otimes^{m} \mathbf{C}^{d}$. It is evident that $U \otimes T=\mathcal{P}_{\zeta}(T \otimes U)$, where $\zeta \in(\ell+\boldsymbol{m})$ ! is the permutation such that $\zeta(1):=m+1, \ldots, \zeta(\ell):=m+\ell, \zeta(\ell+1):=1, \ldots, \zeta(\ell+m):=m$. Therefore $U \vee T=\mathcal{S P}_{\zeta}(T \otimes U)=\mathcal{S}(T \otimes U)=T \vee U\left(\mathcal{P}_{\zeta}\right.$ disappears due to Eq. (A.2)).

Now, let us consider a third tensor $V \in \otimes^{p} \mathbf{C}^{d}$; then $(T \vee U) \vee V$ $=\mathcal{S}(\mathcal{S}(T \otimes U) \otimes V)=\mathcal{S}((T \otimes U) \otimes V))$ (the first passage holds by the definition of $\vee$, the second follows from (A.3)). In a similar way one finds $T \vee(U \vee V)=\mathcal{S}(T \otimes(U \otimes V))$; now, the associativity of $\otimes$ yields the equality $(T \vee U) \vee V=T \vee(U \vee V)$.

Proof of Eq. (3.9) for the tensor norms. Consider tensors $T \in \otimes^{\ell} \mathbf{C}^{d}$, $U \in \otimes{ }^{m} \mathbf{C}^{d}$. The equality $|T \otimes U|^{2}=|T|^{2}|U|^{2}$ is checked in an elementary way, explicitating the definitions of || and $\otimes$. Furthermore,

$$
|T \vee U|=|\mathcal{S}(T \otimes U)| \leq \frac{1}{(\ell+m) !} \sum_{\sigma \in(\ell+m) !}\left|\mathcal{P}_{\sigma}(T \otimes U)\right|,
$$


the last passage following from the definition of $\mathcal{S}$ and the triangular inequality for || . On the other hand, it is clear from the very definition that each operator $\mathcal{P}_{\sigma}$ is isometric w.r.t. the norm || ; so $\left|\mathcal{P}_{\sigma}(T \otimes U)\right|$ $=|T \otimes U|=|T||U|$ for all $\sigma \in(\ell+m)$ !, and this implies $|T \vee U| \leq|T||U|$, as desired.

Identities for the derivative $\nabla$ and the operators $\mathcal{P}_{\sigma}, \mathcal{S}$. We regard all these operators as acting on the $C^{1}$ tensor fields of some definite order. For each order $\ell$ and each permutation $\sigma \in \ell$ !, it is

$$
\nabla \mathcal{P}_{\sigma}=\mathcal{P}_{\widetilde{\sigma}} \nabla
$$

where $\widetilde{\sigma} \in(\ell+1)$ ! is defined by $\widetilde{\sigma}(i):=\sigma(i)$ for $1 \leq i \leq \ell, \widetilde{\sigma}(\ell+1):=\ell+1$; this fact is straightforwardly checked using the definition (3.12) of $\nabla$.

As a second fact, on tensor fields of any order $\ell$ we have the identity

$$
\mathcal{S} \nabla \mathcal{S}=\mathcal{S} \nabla
$$

this follows writing $\mathcal{S} \nabla \mathcal{S}=(1 / \ell !) \sum_{\sigma \in \ell} ! \mathcal{S} \nabla \mathcal{P}_{\sigma}$, and using Eqs. (A.4) (A.2).

Leibnitz rule for $\nabla$ and $\otimes$. For all $C^{1}$ tensor fields $T: \mathbf{R}^{d} \rightarrow \otimes^{\ell} \mathbf{C}^{d}$ and $U: \mathbf{R}^{d} \rightarrow \otimes^{m} \mathbf{C}^{d}$, it is

$$
\nabla(T \otimes U)=\mathcal{P}_{\eta}(\nabla T \otimes U)+T \otimes \nabla U,
$$

where $\eta \in(\ell+\boldsymbol{m}+\mathbf{1})$ ! is defined by $\eta(1):=1, \ldots, \eta(\ell):=\ell, \eta(\ell+1):=$ $\ell+m+1, \ldots, \eta(\ell+2):=\ell+1, \eta(\ell+3):=\ell+2, \ldots, \eta(\ell+m+1):=\ell+m$. To prove this we note that, in terms of components, we have

$$
\begin{gathered}
(\nabla(T \otimes U))_{\lambda_{1} \ldots \lambda_{\ell+m+1}}=\partial_{\lambda_{\ell+m+1}}\left(T_{\lambda_{1} \ldots \lambda_{\ell}} U_{\lambda_{\ell+1} \ldots \lambda_{\ell+m}}\right)= \\
=\left(\partial_{\lambda_{\ell+m+1}} T_{\lambda_{1} \ldots \lambda_{\ell}}\right) U_{\lambda_{\ell+1} \ldots \lambda_{\ell+m}}+T_{\lambda_{1} \ldots \lambda_{\ell}}\left(\partial_{\ell+m+1} U_{\lambda_{\ell+1} \ldots \lambda_{\ell+m}}\right) ;
\end{gathered}
$$

Eq. (A.6) is just a compact formulation of this result.

"Symmetrized" Leibnitz rule for $\nabla_{S}$ and $\vee$. This is given by Eq. (3.16), here reported:

$$
\nabla_{\mathcal{S}}(T \vee U)=\left(\nabla_{\mathcal{S}} T\right) \vee U+T \vee\left(\nabla_{\mathcal{S}} U\right)
$$


for all $C^{1}$ tensor fields $T, U$ of arbitrary orders $\ell, m$. Eq. (3.16) is proved by the chain of relations

$$
\begin{aligned}
& \nabla_{\mathcal{S}}(T \vee U) \stackrel{\text { def.s of }}{=} \nabla_{\mathcal{S}}, \vee \mathcal{S} \nabla \mathcal{S}(T \otimes U) \\
& \stackrel{(\text { A.5) }}{=} \mathcal{S} \nabla(T \otimes U) \\
& \stackrel{(\text { A.6) }}{=} \mathcal{S} \mathcal{P}_{\eta}(\nabla T \otimes U)+\mathcal{S}(T \otimes \nabla U) \\
& \stackrel{(\text { A.2) }}{=} \mathcal{S}(\nabla T \otimes U)+\mathcal{S}(T \otimes \nabla U) \\
& \stackrel{(\text { A.3) }}{=} \mathcal{S}(\mathcal{S} \nabla T \otimes U)+\mathcal{S}(T \otimes \mathcal{S} \nabla U) \\
& \text { def.s } \stackrel{\text { of }}{=} \nabla_{\mathcal{S}}, \vee \nabla_{\mathcal{S}} T \vee U+T \vee \nabla_{\mathcal{S}} U .
\end{aligned}
$$

\section{References}

[1] D.R. Adams, On the existence of capacitary strong type estimates in $\boldsymbol{R}^{n}$, Ark. Math., 14 (1976), 125-140; D.R. Adams, M. Frazier, BMO and smooth truncation in Sobolev spaces, Studia Math., 39 (1988), 241-260, Composition operators on potential spaces, Proc. AMS, 114 (1992), 155-165.

[2] N. Aronszajn and K.T. Smith, Theory of Bessel potentials, I, Ann. Inst. Fourier, 11 (1961), 385-475.

[3] W. Beckner, Inequalities in Fourier Analysis, Ann. of Math., 102 (1975), 159-182.

[4] G. Bourdaud, Le calcul fonctionnel dans les espaces de Sobolev, Invent. Math., 104 (1991), 435-446 ; The functional calculus in Sobolev spaces, in Proc. Conf. 'Function spaces, differential operators and nonlinear analysis', Teubner-Text Math., 133 (1993), 127-142, Teubner, Leipzig.

[5] L. Comtet, Advanced Combinatorics, Reidel, Dordrecht, 1974.

[6] A. Friedman, Partial Differential Equations, Holt, Rinehart and Winston, New York, 1969.

[7] J. Garcia-Cuerva and J.L. Rubio de Francia, Weighted Norm Inequalities and Related Topics, Mathematics Studies, 116 (1985), North-Holland, Amsterdam.

[8] H. Gzyl, Multidimensional extension of Fà̀ di Bruno's formula, J. Math. Anal. Appl., 116 (1986), 450-455.

[9] R.S. Hamilton, The inverse function theorem of Nash and Moser, Bull. Amer. Math. Soc. (N.S.), 7 (1982), 65-222. 
[10] V. Kac, Infinite Dimensional Lie Algebras, Cambridge Univ. Press, Cambridge, 1990.

[11] A. I. Kostrikin and Y.I. Manin, Linear Algebra and Geometry, Gordon and Breach, New York, 1989.

[12] E. H. Lieb and M. Loss, Analysis, Graduate Studies in Mathematics, 14 (1997), American Mathematical Society.

[13] V.G. Maz’ja, Sobolev Spaces, Springer, Berlin, 1985.

[14] G. Meloni, Private communication.

[15] R. L. Mishkov, Generalization of the formula of Fà̀ di Bruno for a composite function with a vector argument, Int. J. Math. Math. Sci., 24 (2000), 481-491.

[16] C. Morosi and L. Pizzocchero, Semilinear evolution equations in Fréchet spaces. Abstract theory, Quaderno 23 (1999), Dipartimento di Matematica, Università di Milano.

[17] C. Morosi and L. Pizzocchero, On the constants for some Sobolev imbeddings, J. Inequal. Appl., 6 (2001), 665-679.

[18] C. Morosi and L. Pizzocchero, On the constants in some inequalities for the Sobolev norms and pointwise product, J. Inequal. Appl., 7 (2002), 421-452.

[19] T. Runst and W. Sickel, Sobolev spaces of fractional order, Nemytskij operators and nonlinear partial differential equations, de Gruyter, Berlin, 1996.

[20] S. Sternberg, Lectures on Differential Geometry, Chelsea, New York, 1983.

[21] K. Yosida, Functional Analysis, Springer, Berlin, 1965.

Dipartimento di Matematica

Politecnico di Milano

P.za L. da Vinci 32, I-20133 Milano

Italy

(E-mail : carmor@mate.polimi.it)

Dipartimento di Matematica

Università di Milano

Via C. Saldini 50, I-20133 Milano

Italy

and

Istituto Nazionale di Fisica Nucleare

Sezione di Milano

Italy

(E-mail : livio.pizzocchero@mat.unimi.it)

(Received : June 2003) 


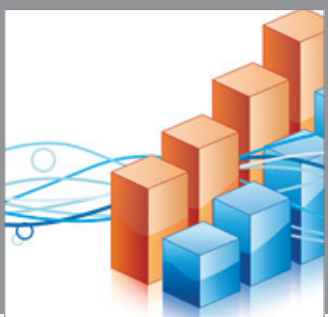

Advances in

Operations Research

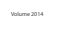

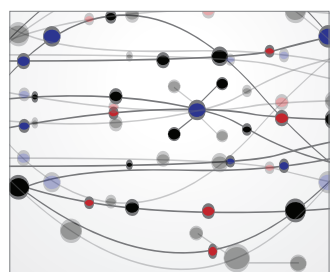

\section{The Scientific} World Journal
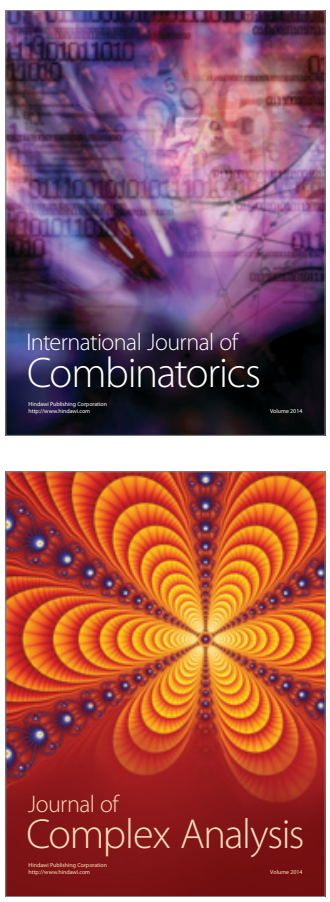

International Journal of

Mathematics and

Mathematical

Sciences
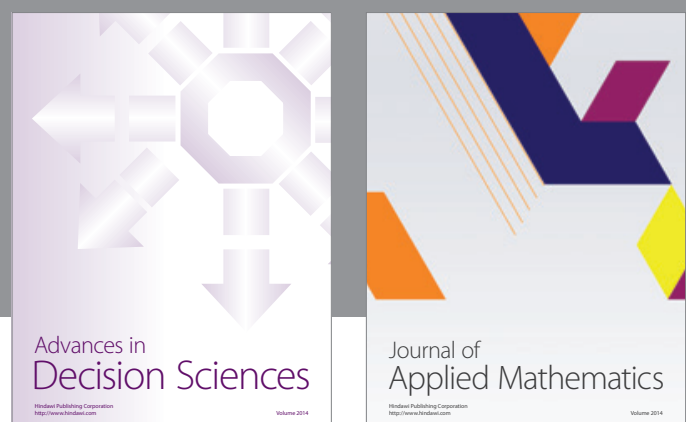

Journal of

Applied Mathematics
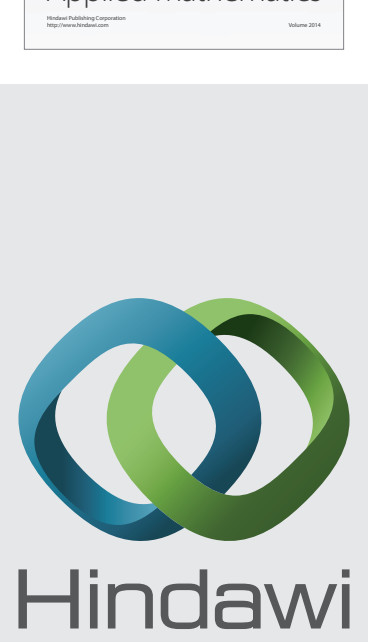

Submit your manuscripts at http://www.hindawi.com
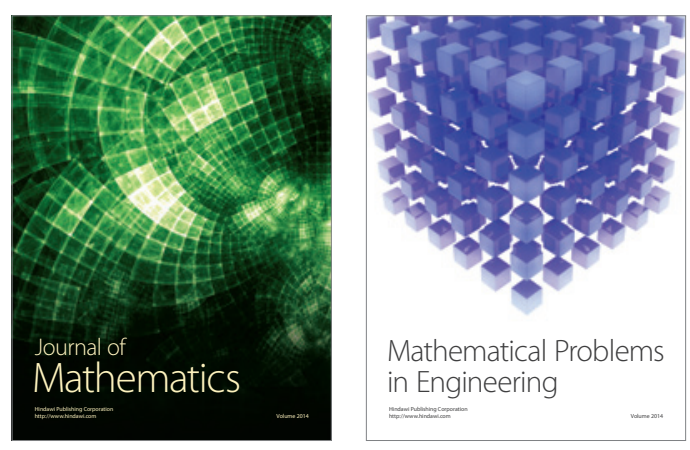

Mathematical Problems in Engineering
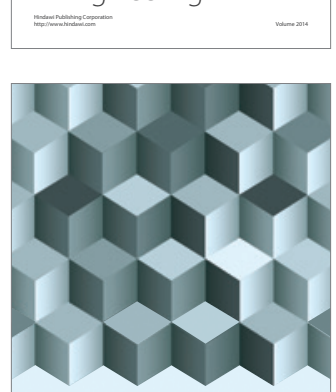

Journal of

Function Spaces
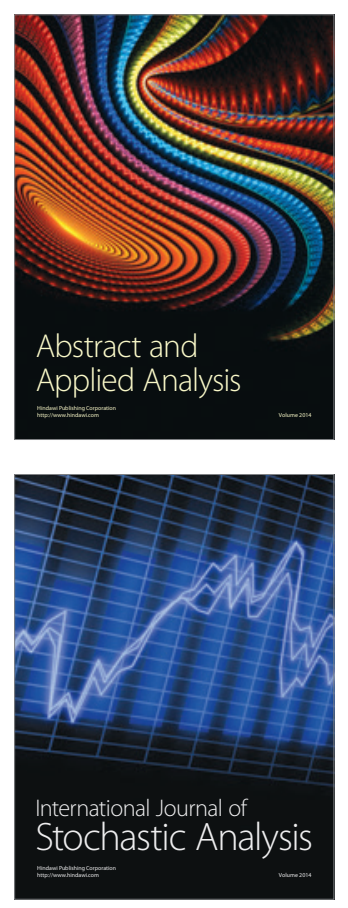

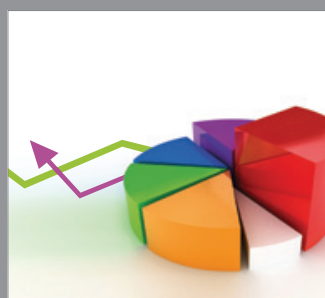

ournal of

Probability and Statistics

Promensencen
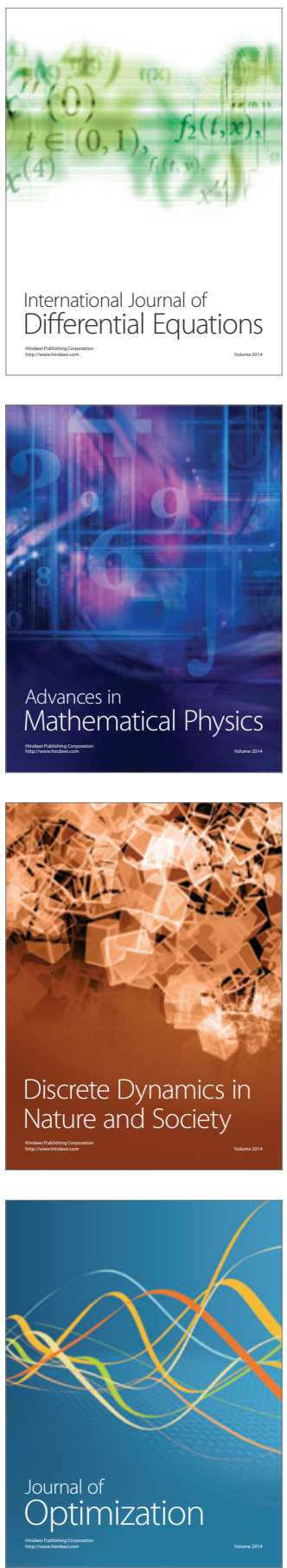